\title{
Review
}

Drug/Regimen

Diabetes Metab J 2020;44:11-32

https://doi.org/10.4093/dmj.2019.0208

pISSN 2233-6079 · eISSN 2233-6087

DIABET\&S \& METABOLISM JOURNAL

\section{Use of SGLT-2 Inhibitors in Patients with Type 2 Diabetes Mellitus and Abdominal Obesity: An Asian Perspective and Expert Recommendations}

\author{
Wayne Huey-Herng Sheu ${ }^{1}$, Siew Pheng Chan ${ }^{2}$, Bien J. Matawaran ${ }^{3}$, Chaicharn Deerochanawong ${ }^{4}$, Ambrish Mithal ${ }^{5}$, \\ Juliana Chan ${ }^{6}$, Ketut Suastika ${ }^{7}$, Chin Meng Khoo ${ }^{8}$, Huu Man Nguyen', Ji Linong ${ }^{10}$, Andrea Luk ${ }^{6}$, Kun-Ho Yoon ${ }^{11}$ \\ ${ }^{1}$ Division of Endocrinology and Metabolism, Department of Internal Medicine, Taichung Veterans General Hospital, Taichung, Taiwan, \\ ${ }^{2}$ Department of Medicine, University of Malaya Medical Centre, Kuala Lumpur, Malaysia, \\ ${ }^{3}$ Section of Endocrinology, Diabetes and Metabolism, Department of Medicine, University of Santo Tomas Hospital, Manila, Philippines, \\ ${ }^{4}$ Department of Internal Medicine, Rajavithi Hospital, College of Medicine, Rangsit University, Bangkok, Thailand, \\ ${ }^{5}$ Division of Endocrinology and Diabetes, The Medicity, Gurgaon, India, \\ ${ }^{6}$ Department of Medicine and Therapeutics, The Chinese University of Hong Kong, Hong Kong, \\ ${ }^{7}$ Department of Internal Medicine, Sanglah General Hospital, Faculty of Medicine, Udayana University, Bali, Indonesia, \\ ${ }^{8}$ Department of Medicine, National University Health System, Singapore, \\ ${ }^{9}$ The Hospital for Tropical Diseases, Ho Chi Minh City, Vietnam, \\ ${ }^{10}$ Department of Endocrinology and Metabolism, Peking University People’s Hospital, Peking, China, \\ ${ }^{11}$ Department of Endocrinology and Metabolism, College of Medicine, The Catholic University of Korea, Seoul, Korea
}

The prevalence of obesity in Asia is of epidemic proportions, with an estimated 1 billion overweight/obese individuals in the region. The majority of patients with type 2 diabetes mellitus (T2DM) are overweight/obese, which increases the risk of cardiorenal outcomes in these patients; hence, sustained reductions in body weight and visceral adiposity are important management goals. However, most of the glucose-lowering therapies such as insulin, sulfonylureas, glinides, and thiazolidinediones induce weight gain, which makes the management of overweight/obese T2DM patients challenging. Sodium-glucose cotransporter-2 (SGLT-2) inhibitors are the only oral glucose-lowering agents that have been shown to reduce body weight and visceral adiposity. In addition, SGLT-2 inhibitors therapy reduces ectopic fat deposition and improves adipose tissue function and weight-related quality of life. In this article, we aim to consolidate the existing literature on the effects of SGLT-2 inhibitors in Asian patients with T2DM and to produce clinical recommendations on their use in overweight or obese patients with T2DM. Recommendations from international and regional guidelines, as well as published data from clinical trials in Asian populations and cardiovascular outcomes trials are reviewed. Based on the available data, SGLT-2 inhibitors represent an evidence-based therapeutic option for the management of overweight/obese patients with T2DM.

Keywords: Diabetes mellitus, type 2; Obesity; Sodium-glucose transporter 2 inhibitors

\section{INTRODUCTION}

Obesity is a serious global health concern and is a major contributing factor to the development of type 2 diabetes mellitus (T2DM). The pathophysiology of obesity and T2DM is closely linked with insulin resistance, which plays a key role in the dysregulation of glucose, lipid, and protein metabolism [1]. The chronic positive net energy balance increases adiposity and circulating levels of free fatty acids (FFAs), which accelerate the development of T2DM through impaired hepatic and
Corresponding author: Kun-Ho Yoon (D) https://orcid.org/0000-0002-9109-2208 Department of Endocrinology and Metabolism, Seoul St. Mary’s Hospital, College of Medicine, The Catholic University of Korea, 222 Banpo-daero, Seocho-gu, Seoul 06591, Korea

E-mail: yoonk@catholic.ac.kr

Received: Nov. 5, 2019; Accepted: Jan. 15, 2020
This is an Open Access article distributed under the terms of the Creative Commons Attribution Non-Commercial License (https://creativecommons.org/licenses/by-nc/4.0/) which permits unrestricted non-commercial use, distribution, and reproduction in any medium, provided the original work is properly cited. 
peripheral glucose metabolism, reduced insulin clearance, impaired pancreatic $\beta$-cell function, ectopic fat deposition, and activation of pro-inflammatory pathways [2,3].

The measures of abdominal adiposity, such as waist circumference (WC), correlate with insulin resistance and markers of cardiovascular disease (CVD) better than body mass index (BMI) does $[4,5]$. Asian populations have higher abdominal obesity for the same BMI compared with their Caucasian counterparts, partly explaining the higher insulin resistance and excessive risk of T2DM and CVD among Asian populations [6,7]. Sniderman et al. [8] proposed that the superficial subcutaneous fat compartment is less developed, with a reduced storage capacity, in South Asians compared with Caucasians, which results in the preferential distribution of fat in visceral or deep subcutaneous depots. The underlying reason for this less developed superficial subcutaneous compartment has been suggested to be the heat stress or the chronic energy deficiency from malnutrition in the evolutionary past, resulting in a preference for the highly vascular visceral adipose tissue with high lipolytic activity $[8,9]$. The harmful effects of higher abdominal obesity in Asians are well recognized, and the World Health Organization (WHO)'s expert committee has recommended a lower BMI level as an actionable cutoff for the risk of T2DM and CVD in Asians $\left(23 \mathrm{~kg} / \mathrm{m}^{2}\right.$ vs. $25 \mathrm{~kg} / \mathrm{m}^{2}$ in Caucasians) [10]. For WC, the WHO provided gender-specific cutoff points for the risk of metabolic complications: $94 \mathrm{~cm}$ (men) and $80 \mathrm{~cm}$ (women) for increased risk, and $102 \mathrm{~cm}$ (men) and $88 \mathrm{~cm}$ (women) for the substantially increased risk [11]. Population and geography-specific WC cutoffs are provided by the International Diabetes Federation and these are lower for Asian men compared with their Caucasian counterparts: 90 $\mathrm{cm}$ (men) and $80 \mathrm{~cm}$ (women) for South Asian, Chinese, and Japanese populations versus $94 \mathrm{~cm}$ (men) and $80 \mathrm{~cm}$ (women) for Caucasians [12]. In Korea, the appropriate WC cutoff for the risk of metabolic syndrome was determined to be $90 \mathrm{~cm}$ in men and $85 \mathrm{~cm}$ in women [13].

It has been well recognized that weight reduction is a key strategy in the management of obese patients with T2DM. However, most of the glucose-lowering therapies, including insulin, sulfonylureas (SUs), glinides, and thiazolidinediones (TZDs), induce weight gain. The recently introduced glucagon-like peptide-1 receptor agonists (GLP-1 RAs) and sodium-glucose cotransporter-2 (SGLT-2) inhibitors have been shown to reduce body weight and have become the preferred agents for the management of overweight/obese patients with
T2DM [14,15]. Previously, we reviewed the effects of SGLT-2 inhibitors on various cardiovascular (CV) risk factors, and developed expert recommendations on the clinical use of SGLT2 inhibitors in Asian T2DM patients with CVD or multiple risk factors [16]. In the present article, we aim to review and consolidate the existing literature on the effects of SGLT-2 inhibitors on body weight and adiposity in Asian patients with T2DM. We then produced a series of clinical recommendations on the use of SGLT-2 inhibitors in overweight/obese patients with T2DM.

\section{EPIDEMIOLOGY AND ASSOCIATED COMPLICATIONS OF OVERWEIGHT/ OBESITY AND T2DM IN ASIA}

The worldwide prevalence of overweight adults aged $>18$ years is estimated to be about 1.9 billion ( $39 \%$ of adults worldwide), and that of obesity is approximately 650 million (13\% of adults worldwide) $[17,18]$. Data from the Global Burden of Disease Study show that high BMI accounts for 4 million deaths annually, of which more than two-thirds are due to CVD [19]. In addition, high BMI contributes to 120 million disability-adjusted life-years. Of note, about $34 \%$ of high BMI-related deaths and $32 \%$ of disability-adjusted life-years occur in individuals with BMI 25 to $30 \mathrm{~kg} / \mathrm{m}^{2}$.

In the Asia Pacific region, an estimated 1 billion people (40.9\%) are overweight/obese (2013 data; using BMI cutoff of $25 \mathrm{~kg} / \mathrm{m}^{2}$ for overweight and $30 \mathrm{~kg} / \mathrm{m}^{2}$ for obesity) [20]. The Global Burden of Disease Study reported region-wise prevalence estimates for overweight and obese adults. Within Asia Pacific, the prevalence of overweight and obesity is as follows: Australasia 68.6\% (men) and 56.7\% (women), East Asia 28.0\% (men) and 27.1\% (women), Southeast Asia 22.1\% (men) and 28.3\% (women), South Asia 20.2\% (men) and 22.5\% (women), and high-income Asian countries (Brunei, Japan, Singapore, and South Korea) $31.7 \%$ (men) and 20.6\% (women) [19]. Also, the region accounts for about $56.7 \%$ of the global diabetes prevalence, with 158.8 million (9.5\%) and 82 million (8.5\%) adults with diabetes in the Western Pacific and Southeast Asia regions, respectively [21]. Over $85 \%$ of all T2DM patients are either overweight or obese, rendering a vast majority of T2DM patients at high risk of CV morbidity and mortality [22]. In the Joint Asia Diabetes Evaluation (JADE) program involving 3,687 patients from nine Asian countries, 30.1\% (range, 20.8\% to $38.7 \%$ ) of patients with T2DM had a BMI of $\geq 27.5 \mathrm{~kg} / \mathrm{m}^{2}$ 
and $53.5 \%$ (range, $19.4 \%$ to $76.4 \%$ ) had abdominal obesity (WC $\geq 80 \mathrm{~cm}$ in women or $\geq 90 \mathrm{~cm}$ in men) [23]. Data from the Korea National Health and Nutrition Examination Survey, a nationally representative survey of the Korean population, showed that $50.4 \%$ of Korean patients with T2DM were obese (BMI $\geq 25 \mathrm{~kg} / \mathrm{m}^{2}$ ) and $47.8 \%$ have abdominal obesity (WC $\geq 85 \mathrm{~cm}$ in women or $\geq 90 \mathrm{~cm}$ in men) [24].

Obesity increases the risk of hypertension, dyslipidemia, and coronary heart diseases in patients with T2DM. In addition, obesity and T2DM are chronic inflammatory states that further increase the risk of $\mathrm{CV}$ and cerebrovascular complications [25-27]. A pooled data analysis of 33 cohort studies ( $n=$ 310,283; mean follow-up 6.9 years) by the Asia Pacific Cohort Studies Collaboration (APCSC) showed that each 2-units' decrease in BMI is associated with a $12 \%$ lower risk of ischemic stroke and $11 \%$ lower risk of ischemic heart disease [28]. Another pooled data analysis by APCSC involving six cohort studies in adults aged $\geq 20$ years $(n=45,988$; mean follow-up 6.1 years) revealed that each standard deviation increase in BMI, WC, and the waist-to-hip ratio increases the risk of ischemic heart disease by $17 \%$ to $36 \%$ [29]. Also, metabolic syndrome is independently associated with ischemic heart disease and ischemic stroke [30].

A U-shaped relationship has been reported between BMI and mortality due to heart failure (HF), with a $>60 \%$ higher risk of HF mortality in both underweight and obese individuals compared with healthy-weight individuals [31]. A pooled analysis of the Framingham Heart, Framingham Offspring, Chicago Heart Association Detection Project in Industry, and Atherosclerosis Risk In Communities (ARIC) studies evaluated the risk of incident HF in individuals with or without hypertension, obesity, or diabetes. Men and women without hypertension, obesity, or diabetes at the age of 45 years have $73 \%$ and $85 \%$ lower risk of incident HF compared with those with all three risk factors, respectively. In addition, individuals without any of the three risk factors at the age of 45 years live 3 to 15 years longer, free of HF, than those with one, two, or all three risk factors [32].

Furthermore, obesity is independently associated with the development and progression of chronic kidney disease (CKD) [33]. Results from the Renal and Vascular End-Stage Disease (PREVEND) study showed that obesity and central fat distribution are associated with a 1.7-fold higher risk of microalbuminuria and 2.7-fold higher risk of decreased glomerular filtration rate [34]. Similarly, a multinational, observational study of 20,828 outpatients with hypertension from 26 countries in Europe, North and Latin America, the Middle East, and Asia, showed that abnormal WC is associated with microalbuminuria, independent of BMI [35]. A retrospective analysis of National Diabetes Audit data (United Kingdom) showed that patients with T2DM and severe kidney disease (estimated glomerular filtration rate [eGFR] $<30 \mathrm{~mL} / \mathrm{min} / 1.73 \mathrm{~m}^{2}$ ) are up to 1.75 times more likely to be obese compared with those with normal kidney function [36]. Furthermore, the risk of CKD (eGFR $<60 \mathrm{~mL} / \mathrm{min} / 1.73 \mathrm{~m}^{2}$ ) is increased by $55 \%$ in the presence of metabolic syndrome [37].

The chronic elevation of circulatory FFAs and reduced FFA oxidation in obese individuals lead to ectopic fat deposition in non-adipose tissues, such as liver, skeletal muscle, heart, and pancreatic $\beta$-cells. Non-alcoholic fatty liver disease (NAFLD) is frequently reported in obese T2DM patients, which may increase the risk of non-alcoholic steatohepatitis, cirrhosis, and hepatocellular cancer [38].

There is a positive association between the presence of diabetes and high BMI $\left(>25 \mathrm{~kg} / \mathrm{m}^{2}\right)$ and the incidence of cancer. Pearson-Stuttard et al. [39] analyzed the incidence of malignancies attributable to diabetes and high BMI as a combined risk factor, across 175 countries. Overall, 5.7\% of all incident cancers are attributable to the combined effects of diabetes and high BMI as independent risk factors [39]. Of these 804,100 cancer cases, 23.8\% occurred in East and Southeast Asia. Among the specific cancer types, $24.5 \%$ of liver cancer and $38.4 \%$ of endometrial cancer cases are attributable to these risk factors [39].

Furthermore, obstructive sleep apnea is prevalent in patients with abdominal obesity and may adversely affect glucose control in patients with T2DM [40].

\section{CURRENT GUIDELINES FOR THE MANAGEMENT OF OVERWEIGHT/OBESE PATIENTS WITH T2DM}

The clinical practice guidelines from Asian countries for the management of T2DM recommend a weight loss goal of 5\% to $10 \%$ to improve glycemic control, blood pressure, lipid profile and quality of life (QoL) in overweight/obese patients with T2DM [41-45]. Similarly, guidelines from Western countries recommend a weight loss goal of $5 \%$ to $15 \%$ in these patients $[14,46,47]$.

For the management of hyperglycemia in overweight/obese 
patients with T2DM, the guidelines recommend the use of glucose-lowering drugs (GLDs) that promote weight loss or that are weight-neutral $[15,48]$. Insulin secretagogues, such as SUs, and glinides have been shown to increase body weight in T2DM patients $[49,50]$. TZDs are associated with weight gain in the range of $3.6 \mathrm{~kg}$ (at 3 years) to $4.8 \mathrm{~kg}$ (at 5 years) in large randomized controlled trials [51,52]. Several meta-analyses investigating the effect of oral GLDs on body weight showed that the addition of SUs, TZDs, and glinides to metformin is associated with significant weight gain in patients with T2DM (range, 1 to $5 \mathrm{~kg}$ ) $[53,54]$. Conversely, metformin, $\alpha$-glucosidase inhibitors, and dipeptidyl peptidase-4 (DPP-4) inhibitors have a neutral effect on body weight [54-56]. Currently, GLDs that promote weight loss include SGLT-2 inhibitors and GLP-1 RAs. The 2018 guidelines from the American Diabetes Association and the European Association for the Study of Diabetes provided a treatment algorithm for patients with a compelling need to minimize weight gain or promote weight loss, and recommended either GLP-1 RAs or SGLT-2 inhibitors as the preferred choice of therapy after metformin [57].

\section{Methods}

An expert panel comprising 12 endocrinology experts from China, Hong Kong, India, Indonesia, Malaysia, the Philippines, Singapore, South Korea, Taiwan, Thailand, and Vietnam met four times (Bangkok, November 2017; Shanghai, March 2018; Orlando, June 2018; Kuala Lumpur, November 2018) to review the clinical evidence and develop clinical recommendations on the use of SGLT-2 inhibitors in overweight/obese Asian patients with T2DM. A literature search was conducted in the MEDLINE database using the search string: ("canagliflozin" OR "dapagliflozin” OR "empagliflozin” OR “ipragliflozin" OR "luseogliflozin" OR "tofogliflozin”) AND "type 2 diabetes." The panel critically analyzed the studies of SGLT-2 inhibitors conducted in Asia as well as recommendations from international and regional guidelines. Following discussions, the group reached consensus on a series of recommendations supported by scientific evidence and experts' clinical opinion.

\section{ROLE OF SGLT-2 INHIBITORS IN MANAGING OVERWEIGHT/OBESE PATIENTS WITH T2DM: AN ASIAN PERSPECTIVE}

SGLT-2 inhibitors selectively and reversibly inhibit SGLT-2 transporters in the proximal convoluted tubule, preventing the renal reabsorption of glucose, thereby increasing urinary glucose excretion. The glucose-lowering effects of SGLT-2 inhibitors are independent of insulin function; hence, they are effective at all stages of T2DM [58]. The glycemic efficacy and safety of SGLT-2 inhibitors as monotherapy or in combination with other GLDs are well established. Results from three CV outcomes trials (EMPA-REG OUTCOME, CANVAS, DECLARETIMI 58) showed beneficial effects of SGLT-2 inhibitors in reducing the risk of CV outcomes in patients with T2DM [59-61].

In the DECLARE-TIMI 58 trial, dapagliflozin treatment reduced the body weight by $-1.8 \mathrm{~kg}$ compared with placebo at the median follow-up of 4.2 years [60]. Similar results are reported with empagliflozin and canagliflozin in the EMPAREG OUTCOME and CANVAS trials respectively [59,61]. In all three cardiovascular outcome trials (CVOTs) the beneficial CV effects of SGLT-2 inhibitors were consistent across BMI subgroups ( $<30$ or $\geq 30 \mathrm{~kg} / \mathrm{m}^{2}$ ). Furthermore, in a meta-analysis of 55 randomized controlled trials, SGLT-2 inhibitor treatment was associated with a consistent and significant reduction in body weight with a dose-dependent response observed for dapagliflozin [62]. A recent, single-center real-world study from India including 486 patients with T2DM reported a mean weight loss of 3.2 and $3.9 \mathrm{~kg}$ with SGLT-2 inhibitor therapy at 6 and 12 months, respectively [63].

These effects of SGLT-2 inhibitors on body weight are of particular significance for overweight/obese patients with $\mathrm{T} 2 \mathrm{DM}$, in whom consistent weight loss and reduction in fat mass are critical for effective management (Fig. 1). In the following sections, we summarize the effects of SGLT-2 inhibitors on body weight, fat mass, and adipose tissue function in Asian T2DM patients.

\section{Effects on body weight in patients with T2DM}

In Western populations, SGLT-2 inhibitor treatment induces a weight loss as monotherapy (weighted mean difference vs. placebo: $-1.74 \mathrm{~kg}$; $95 \%$ confidence interval $[\mathrm{CI}],-2.03$ to -1.45 ) and as combination therapy (weighted mean difference vs. other GLDs: $-1.80 \mathrm{~kg}$; $95 \%$ CI, -3.50 to $-0.11 \mathrm{~kg}$ ) [64]. Similar results are observed in Asian patients with T2DM. Treatment with SGLT-2 inhibitors resulted in a significant reduction in body weight, ranging from -0.5 to $-3.9 \mathrm{~kg}$ (study duration range, 8 to 104 weeks). The effect of SGLT-2 inhibitors on body weight in Asian patients with T2DM is summarized in Table 1 [65-106]. 


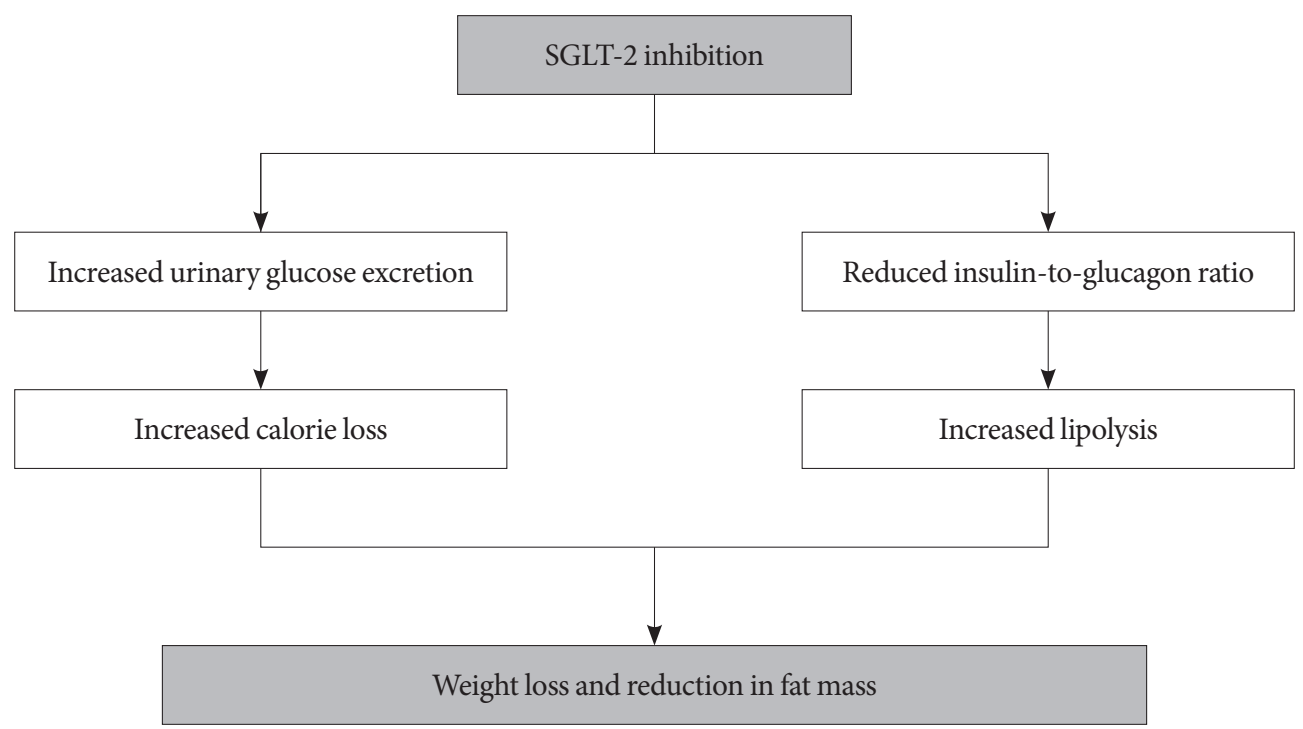

Fig. 1. Proposed mechanisms of effects of sodium-glucose cotransporter-2 (SGLT-2) inhibitors on body weight and fat mass.

As monotherapy in treatment-naïve Asian and Japanese patients with T2DM, consistent reduction in body weight is observed with dapagliflozin (range, -1.24 to $-2.25 \mathrm{~kg}$ ), canagliflozin (range, -1.98 to $-3.19 \mathrm{~kg}$ ), and empagliflozin treatments (range, -2.5 to $-3.1 \mathrm{~kg}$ ) (study duration up to 24 weeks) (Table 1). Similar effects are observed with ipragliflozin, luseogliflozin, and tofogliflozin, with body weight reductions ranging from -1.1 to $-2.97 \mathrm{~kg}$ (study duration of 8 to 52 weeks) (Table 1) [65-67].

The reduction in body weight is also reported when SGLT-2 inhibitors are used in combination with other oral GLDs (range, -1.56 to $-3.9 \mathrm{~kg}$; study duration up to 52 weeks) (Table 1). Two studies reported on the effects of canagliflozin and luseogliflozin as an add-on to GLP-1 RAs in Japanese patients with T2DM. Addition of canagliflozin $100 \mathrm{mg}$ to GLP-1 RAs resulted in a body weight reduction of $-3.29 \mathrm{~kg}(4.46 \%)$ at 52 weeks [68]. Luseogliflozin as an add-on to liraglutide was associated with significant weight loss $(-2.71 \mathrm{~kg})$, with $33.9 \%$ of patients achieving a $\geq 5 \%$ reduction at 52 weeks [69]. Furthermore, the 28-week DURATION-8 trial demonstrated comparable effects of an SGLT-2 inhibitor (dapagliflozin) and GLP-1 RA (exenatide) on body weight ( $-2.22 \mathrm{~kg}$ vs. $-1.56 \mathrm{~kg}$, respectively), whereas an additive effect was observed with combination therapy $(-3.55 \mathrm{~kg})$. As GLP-1 RAs also exert weight-lowering effects, these results indicate additive effects of SGLT-2 inhibitors and GLP-1 RAs on weight reduction.

The weight-lowering effects of SGLT-2 inhibitors are consis- tent even when used as an add-on to insulin therapy. In studies with follow-up of up to 52 weeks, weight reduction with SGLT2 inhibitor treatment ranged from -0.5 to $-3.4 \mathrm{~kg}$ [70].

\section{Effect on abdominal adiposity}

In Western populations, SGLT-2 inhibitor treatment reduces WC by about $-1.20 \mathrm{~cm}$ (weighted mean difference vs. placebo; 95\% CI, -2.00 to -0.43 ) [107]. Similar results are observed in Asian patients with T2DM. Across the studies in patients with T2DM, treatment with SGLT-2 inhibitors reduced WC as monotherapy (range, -1.59 to $-2.82 \mathrm{~cm}$ ), or in combination with other oral GLDs (range, -1.39 to $-3.82 \mathrm{~cm}$ ) or in combination with insulin (range, -0.7 to $-1.4 \mathrm{~cm}$ ) $[65,70,71-78,108]$. Table 1 summarizes the effects of SGLT-2 inhibitors on WC in Asian T2DM patients.

In addition, Ohta et al. [109] evaluated the effects of ipragliflozin on intrahepatic lipid content and subcutaneous and visceral fat volume in Japanese T2DM patients. Treatment with ipragliflozin for 24 weeks was associated with significant reductions in subcutaneous and visceral fat volume, intrahepatic lipid content and fat mass, and appendicular skeletal mass indices. In another 12-month study in obese Japanese T2DM patients $(n=20)$, treatment with ipragliflozin significantly reduced both visceral and subcutaneous fat volume at 3 months; the reduction in subcutaneous fat volume remained significant at 12 months follow-up [110]. The PRIME-V study compared the effects of ipragliflozin and metformin, as an add-on to 
Table 1. Effect of SGLT-2 inhibitors on body weight and waist circumference in Asian patients with T2DM

\begin{tabular}{|c|c|c|c|c|}
\hline Study & Design and population & Intervention & Effect on body weight & Effect on waist circumference \\
\hline \multicolumn{5}{|l|}{ Monotherapy } \\
\hline $\begin{array}{l}\text { Inagaki et al. } \\
(2013)[79]\end{array}$ & $\begin{array}{l}\text { Phase II, } 12-\text { wk } \\
\text { randomized study; } \\
\text { Japanese T2DM } \\
(n=383)\end{array}$ & $\begin{array}{l}\text { Canagliflozin }(50, \\
\text { 100, 200, or } 300 \\
\text { mg); placebo }\end{array}$ & $\begin{array}{l}-1.98 \mathrm{~kg}(50 \mathrm{mg}) ;-2.51 \mathrm{~kg}(100 \\
\mathrm{mg}) ;-2.39 \mathrm{~kg}(200 \mathrm{mg}) ;-3.19 \mathrm{~kg} \\
(300 \mathrm{mg}) ;-0.78 \mathrm{~kg} \text { (placebo) }\end{array}$ & $\begin{array}{l}-1.59 \mathrm{~cm}(50 \mathrm{mg}),-1.81 \mathrm{~cm}(100 \\
\mathrm{mg}),-1.83 \mathrm{~cm}(200 \mathrm{mg}),-2.21 \\
\mathrm{~cm}(300 \mathrm{mg}) ;-0.59 \mathrm{~cm} \text { (placebo) }\end{array}$ \\
\hline $\begin{array}{l}\text { Inagaki et al. } \\
(2014)[73]\end{array}$ & $\begin{array}{l}\text { Phase III, } 24 \text {-wk dou- } \\
\text { ble-blind random- } \\
\text { ized study; Japanese } \\
\text { T2DM }(n=272)\end{array}$ & $\begin{array}{l}\text { Canagliflozin (100, } \\
200 \mathrm{mg}) ; \text { placebo }\end{array}$ & $\begin{array}{l}-3.76 \%(100 \mathrm{mg}),-4.02 \%(200 \\
\mathrm{mg}) ;-0.76 \% \text { (placebo) }\end{array}$ & $\begin{array}{l}-2.21 \mathrm{~cm}(100 \mathrm{mg}) ;-2.82 \mathrm{~cm}(200 \\
\mathrm{mg}) ;-1.03 \mathrm{~cm} \text { (placebo) }\end{array}$ \\
\hline $\begin{array}{l}\text { Kaku et al. } \\
\text { (2013) [80] }\end{array}$ & $\begin{array}{l}\text { Phase II, } 12-\text { wk } \\
\text { randomized study; } \\
\text { Japanese T2DM } \\
(n=279)\end{array}$ & $\begin{array}{l}\text { Dapagliflozin }(1,2.5 \\
\text { 5, or } 10 \mathrm{mg} / \text { day); } \\
\text { placebo }\end{array}$ & $\begin{array}{l}-1.25 \mathrm{~kg}(1 \mathrm{mg}) ;-1.24 \mathrm{~kg}(2.5 \mathrm{mg}) \\
-2.06 \mathrm{~kg}(5 \mathrm{mg}) ;-1.91 \mathrm{~kg}(10 \\
\mathrm{mg}) ;-0.05 \text { (placebo) }\end{array}$ & - \\
\hline $\begin{array}{l}\text { Ji et al. (2014) } \\
\text { [74] }\end{array}$ & $\begin{array}{l}\text { Phase III, 24-wk dou- } \\
\text { ble-blind random- } \\
\text { ized study; drug-na- } \\
\text { ïve Asian T2DM } \\
(n=393)\end{array}$ & $\begin{array}{l}\text { Dapagliflozin ( } 5 \text { or } 10 \\
\text { mg); placebo }\end{array}$ & $\begin{array}{l}-1.64 \mathrm{~kg}(5 \mathrm{mg}) ;-2.25 \mathrm{~kg}(10 \mathrm{mg}) \\
\quad-0.27 \mathrm{~kg} \text { (placebo })\end{array}$ & $\begin{array}{l}-2.77 \mathrm{~cm}(5 \mathrm{mg}) ;-2.20 \mathrm{~cm}(10 \\
\mathrm{mg}) ;-0.72 \mathrm{~cm} \text { (placebo) }\end{array}$ \\
\hline $\begin{array}{l}\text { Kaku et al. } \\
\quad(2014)[81]\end{array}$ & $\begin{array}{l}\text { Phase III, } 24 \text {-wk dou- } \\
\text { ble-blind random- } \\
\text { ized study; Japanese } \\
\text { T2DM }(n=261)\end{array}$ & $\begin{array}{l}\text { Dapagliflozin ( } 5 \text { or } 10 \\
\text { mg); placebo }\end{array}$ & $\begin{array}{l}-2.13 \mathrm{~kg}(5 \mathrm{mg}) ;-2.22 \mathrm{~kg}(10 \mathrm{mg}) \\
\quad-0.84 \mathrm{~kg} \text { (placebo) }\end{array}$ & - \\
\hline $\begin{array}{l}\text { Kadowaki et al. } \\
\text { (2014) [82] }\end{array}$ & $\begin{array}{l}\text { Phase II, 12-wk ran- } \\
\text { domized study; Jap- } \\
\text { anese T2DM } \\
(n=547)\end{array}$ & $\begin{array}{l}\text { Empagliflozin }(5,10 \text {, } \\
25 \text {, or } 50 \mathrm{mg}) \text {; place- } \\
\text { bo }\end{array}$ & $\begin{array}{l}-2.5 \mathrm{~kg}(5 \mathrm{mg}) ;-2.6 \mathrm{~kg}(10 \mathrm{mg}) \\
-2.9 \mathrm{~kg}(25 \mathrm{mg}) ;-3.1 \mathrm{~kg}(50 \mathrm{mg}) \\
-0.9 \mathrm{~kg} \text { (placebo) }\end{array}$ & $\begin{array}{l}-2.4 \mathrm{~cm}(5 \mathrm{mg}) ;-2.3 \mathrm{~cm}(10 \mathrm{mg}) \\
-2.6 \mathrm{~cm}(25 \mathrm{mg}) ;-2.6 \mathrm{~cm}(50 \\
\mathrm{mg}) ;-1.3 \mathrm{~cm} \text { (placebo })\end{array}$ \\
\hline $\begin{array}{l}\text { Kadowaki et al. } \\
\text { (2015) [75] }\end{array}$ & $\begin{array}{l}\text { 40-wk extension } \\
\text { study; Japanese } \\
\text { T2DM }(n=532)\end{array}$ & $\begin{array}{l}\text { Empagliflozin (10, } 25 \\
\text { mg) }\end{array}$ & $-3.1 \mathrm{~kg}(10 \mathrm{mg}) ;-3.1 \mathrm{~kg}(25 \mathrm{mg})$ & $-2.8 \mathrm{~cm}(10 \mathrm{mg}) ;-2.8 \mathrm{~cm}(25 \mathrm{mg})$ \\
\hline $\begin{array}{l}\text { Kashiwagi } \\
\text { et al. (2014) } \\
\text { [66] }\end{array}$ & $\begin{array}{l}\text { Phase II, } 12-\text { wk } \\
\text { randomized study; } \\
\text { Japanese T2DM } \\
(n=361)\end{array}$ & $\begin{array}{l}\text { Ipragliflozin }(12.5,25 \text {, } \\
50 \text {, or } 100 \mathrm{mg} / \text { day }) \\
\text { placebo }\end{array}$ & $\begin{array}{c}-1.46 \mathrm{~kg}(12.5 \mathrm{mg}) ;-1.69 \mathrm{~kg}(25 \\
\mathrm{mg}) ;-1.81 \mathrm{~kg}(50 \mathrm{mg}) ;-2.1 \mathrm{~kg} \\
(100 \mathrm{mg}) ;-0.39 \mathrm{~kg} \text { (placebo) }\end{array}$ & - \\
\hline $\begin{array}{l}\text { Kashiwagi } \\
\text { et al. (2015) } \\
\text { [83] }\end{array}$ & $\begin{array}{l}\text { Phase III, 16-wk dou- } \\
\text { ble-blind random- } \\
\text { ized study; Japanese } \\
\text { T2DM }(n=131)\end{array}$ & $\begin{array}{l}\text { Ipragliflozin }(50 \mathrm{mg}) \\
\text { placebo }\end{array}$ & $\begin{array}{l}-2.31 \mathrm{~kg}(50 \mathrm{mg}) ;-1.03 \mathrm{~kg} \text { (place- } \\
\text { bo) }\end{array}$ & $\begin{array}{l}-1.61 \mathrm{~cm}(50 \mathrm{mg}) ;-0.41 \mathrm{~cm}(\text { pla- } \\
\text { cebo) }\end{array}$ \\
\hline $\begin{array}{l}\text { Seino et al. } \\
\quad(2015)[67]\end{array}$ & $\begin{array}{l}\text { 52-wk open-label } \\
\text { study; Japanese } \\
\text { T2DM }(n=299)\end{array}$ & $\begin{array}{l}\text { Luseogliflozin ( } 2.5 \\
\text { mg, option to upti- } \\
\text { trate to } 5 \mathrm{mg})\end{array}$ & $\begin{array}{l}12 \text { wk: }-1.77 \mathrm{~kg}(2.5 / 5 \mathrm{mg}) \\
24 \text { wk: }-1.91 \mathrm{~kg}(2.5 / 5 \mathrm{mg}) \\
36 \mathrm{wk}:-2.35 \mathrm{~kg}(2.5 / 5 \mathrm{mg}) \\
52 \mathrm{wk}:-2.68 \mathrm{~kg}(2.5 / 5 \mathrm{mg})\end{array}$ & - \\
\hline $\begin{array}{l}\text { Hirose et al. } \\
\text { (2016) [84] }\end{array}$ & $\begin{array}{l}\text { 8-wk open-label study } \\
\quad(n=17)\end{array}$ & Tofogliflozin (20 mg) & $-1.1 \mathrm{~kg}(20 \mathrm{mg})$ & - \\
\hline $\begin{array}{l}\text { Kaku et al. } \\
\text { (2014) [65] }\end{array}$ & $\begin{array}{l}\text { Phase II/III, 24-wk } \\
\text { double-blind } \\
\text { randomized study; } \\
\text { Japanese T2DM } \\
(n=235)\end{array}$ & $\begin{array}{l}\text { Tofogliflozin }(10,20 \text {, } \\
\text { or } 40 \mathrm{mg}) ; \text { placebo }\end{array}$ & $\begin{array}{l}-2.23 \mathrm{~kg}(10 \mathrm{mg}) ;-2.85 \mathrm{~kg}(20 \\
\mathrm{mg}) ;-2.97 \mathrm{~kg}(40 \mathrm{mg}) ;-0.36 \mathrm{~kg} \\
\text { (placebo) }\end{array}$ & $\begin{array}{l}-2.42 \mathrm{~cm}(10 \mathrm{mg}) ;-2.47 \mathrm{~cm}(20 \\
\mathrm{mg}) ;-2.27 \mathrm{~cm}(40 \mathrm{mg}) ; 0.02 \mathrm{~cm} \\
\text { (placebo) }\end{array}$ \\
\hline
\end{tabular}

(Continued to the next page) 
Table 1. Continued

\begin{tabular}{|c|c|c|c|c|}
\hline Study & Design and population & Intervention & Effect on body weight & Effect on waist circumference \\
\hline \multicolumn{5}{|c|}{ Add-on to oral antidiabetic agents } \\
\hline $\begin{array}{l}\text { Ji et al. (2015) } \\
\quad[85]\end{array}$ & $\begin{array}{l}\text { Phase III, 18-wk dou- } \\
\text { ble-blind random- } \\
\text { ized study; Asian } \\
\text { T2DM }(n=678)\end{array}$ & $\begin{array}{l}\text { Canagliflozin (100, } \\
300 \mathrm{mg})+ \text { Met or } \\
\text { SU+Met; placebo }\end{array}$ & $\begin{array}{l}-1.9 \mathrm{~kg}(100 \mathrm{mg}) ;-2.1 \mathrm{~kg}(300 \mathrm{mg}) ; \\
\quad-0.5 \mathrm{~kg} \text { (placebo) }\end{array}$ & - \\
\hline $\begin{array}{l}\text { Inagaki et al. } \\
(2015)[72]\end{array}$ & $\begin{array}{l}\text { 52-wk open-label } \\
\text { randomized study; } \\
\text { Japanese T2DM } \\
(n=1,299)\end{array}$ & $\begin{array}{l}\text { Canagliflozin (100, } \\
200 \mathrm{mg}) \text {; other } \\
\text { GLDs }\end{array}$ & $\begin{array}{l}-4.42 \%(100 \mathrm{mg}) ;-4.70 \%(200 \\
\mathrm{mg}) ;-2.94 \%(100 \mathrm{mg}+\mathrm{SU}) \\
-3.51 \%(200 \mathrm{mg}+\mathrm{SU}) ;-3.97 \% \\
\text { (100 mg+glinide); }-4.37 \%(200 \\
\text { mg+glinide); }-4.03 \%(100 \mathrm{mg}+\alpha- \\
\mathrm{GI}) ;-4.98 \%(200 \mathrm{mg}+\alpha-\mathrm{GI}) ; \\
-4.42 \%(100 \mathrm{mg}+\mathrm{BG}) ;-5.54 \% \\
\text { (200 mg+BG);-3.37\% (100 } \\
\text { mg+TZD); }-3.43 \%(200 \\
\text { mg+TZD); }-4.00 \%(100 \\
\text { mg+DPP-4i); }-4.37 \%(200 \\
\text { mg+DPP-4i) }\end{array}$ & $\begin{array}{l}-2.76 \mathrm{~cm}(100 \mathrm{mg}) ;-3.34 \mathrm{~cm}(200 \\
\mathrm{mg}) ;-1.96 \mathrm{~cm}(100 \mathrm{mg}+\mathrm{SU}) ; \\
-1.72 \mathrm{~cm}(200 \mathrm{mg}+\mathrm{SU}) ;-2.93 \mathrm{~cm} \\
(100 \mathrm{mg}+\text { glinide }) ;-3.24 \mathrm{~cm}(200 \\
\text { mg+glinide); }-2.27 \mathrm{~cm}(100 \\
\text { mg+a-GI); -2.57 (200 mg+a-GI); } \\
-3.40 \mathrm{~cm}(100 \mathrm{mg}+\mathrm{BG}) ;-3.82 \\
\mathrm{~cm}(200 \mathrm{mg}+\mathrm{BG}) ;-3.12 \mathrm{~cm}(100 \\
\mathrm{mg}+\mathrm{TZD}) ;-2.64 \mathrm{~cm}(200 \\
\mathrm{mg}+\mathrm{TZD}) ;-3.34 \mathrm{~cm}(100 \\
\mathrm{mg}+\mathrm{DPP}-4 \mathrm{i}) ;-2.37 \mathrm{~cm}(200 \\
\mathrm{mg}+\mathrm{DPP}-4 \mathrm{i})\end{array}$ \\
\hline $\begin{array}{l}\text { Kadowaki et al. } \\
\text { (2017) [86] }\end{array}$ & $\begin{array}{l}\text { Phase III, 24-wk dou- } \\
\text { ble-blind random- } \\
\text { ized study; Japanese } \\
\text { T2DM }(n=138)\end{array}$ & $\begin{array}{l}\text { Canagliflozin (100 } \\
\text { mg); teneligliptin; } \\
\text { placebo }\end{array}$ & $\begin{array}{l}-2.29 \mathrm{~kg} \text { (100 mg+teneligliptin); } \\
-0.78 \mathrm{~kg} \text { (placebo+teneligliptin) }\end{array}$ & - \\
\hline $\begin{array}{l}\text { Kadowaki et al. } \\
\text { (2018) [87] }\end{array}$ & $\begin{array}{l}\text { 52-wk open-label } \\
\text { study; Japanese } \\
\text { T2DM }(n=153)\end{array}$ & $\begin{array}{l}\text { Canagliflozin (100 } \\
\text { mg); teneligliptin }\end{array}$ & -2.86 kg (100 mg+teneligliptin) & - \\
\hline $\begin{array}{l}\text { Harashima } \\
\text { et al. (2018) } \\
\text { [68] }\end{array}$ & $\begin{array}{l}\text { Phase IV, } 52 \text {-wk } \\
\text { open-label; Japanese } \\
\text { T2DM }(n=71)\end{array}$ & $\begin{array}{l}\text { Canagliflozin (100 } \\
\text { mg); liraglutide }\end{array}$ & -3.29 kg (100 mg+liraglutide) & $-3.39 \mathrm{~cm}$ (100 mg+liraglutide) \\
\hline $\begin{array}{l}\text { Kaku et al. } \\
\text { (2014) [81] }\end{array}$ & $\begin{array}{l}\text { Phase III, 52-wk } \\
\text { open-label study; } \\
\text { Japanese T2DM } \\
(n=728)\end{array}$ & $\begin{array}{l}\text { Dapagliflozin ( } 5 \mathrm{mg} \text {, } \\
\text { option to uptitrate } \\
\text { to } 10 \mathrm{mg} \text { ); other } \\
\text { GLDs }\end{array}$ & $\begin{array}{l}-2.6 \mathrm{~kg} \text { (monotherapy); }-2.1 \mathrm{~kg} \\
\text { (combination therapy) }\end{array}$ & $\begin{array}{l}-2.1 \mathrm{~cm} \text { (monotherapy); }-2.0 \mathrm{~cm} \\
\text { (combination therapy) }\end{array}$ \\
\hline $\begin{array}{l}\text { Yang et al. } \\
\text { (2016) [78] }\end{array}$ & $\begin{array}{l}\text { Phase III, 24-wk ran- } \\
\text { domized double- } \\
\text { blind study; Asian } \\
\text { T2DM }(n=444)\end{array}$ & $\begin{array}{l}\text { Dapagliflozin }(5,10 \\
\text { mg); Met; placebo }\end{array}$ & $\begin{array}{l}-1.8 \mathrm{~kg}(5 \mathrm{mg}+\mathrm{Met}) ;-2.6 \mathrm{~kg}(10 \\
\mathrm{mg}+\mathrm{Met}) ;-0.7 \mathrm{~kg} \text { (placebo+Met) }\end{array}$ & $\begin{array}{l}-1.96 \mathrm{~cm}(5 \mathrm{mg}+\mathrm{Met}) ;-2.15 \mathrm{~cm} \\
\quad(10 \mathrm{mg}+\mathrm{Met}) ;-0.39 \mathrm{~cm} \\
\text { (placebo+Met) }\end{array}$ \\
\hline $\begin{array}{l}\text { Araki et al. } \\
\text { (2015) [88] }\end{array}$ & $\begin{array}{l}\text { Phase III, 52-wk } \\
\text { randomized study; } \\
\text { Japanese T2DM } \\
(n=1,160)\end{array}$ & $\begin{array}{l}\text { Empagliflozin (10, } 25 \\
\text { mg); other GLDs }\end{array}$ & $\begin{array}{l}\text { SU: }-2.3 \mathrm{~kg}(10 \mathrm{mg}+\mathrm{SU}) ;-2.8 \mathrm{~kg} \\
\quad(25 \mathrm{mg}+\mathrm{SU}) \\
\text { BG: }-3.9 \mathrm{~kg}(10 \mathrm{mg}+\mathrm{BG}) ;-3.4 \mathrm{~kg} \\
(25 \mathrm{mg}+\mathrm{BG}) \\
\text { TZD: }-2.6 \mathrm{~kg}(10 \mathrm{mg}+\mathrm{TZD}) ;-2.8 \\
\text { kg (25 mg+TZD) } \\
\text { a-GI: }-3.8 \mathrm{~kg}(10 \mathrm{mg}+\mathrm{\alpha}-\mathrm{GI}) ;-3.4 \\
\text { kg (25 mg+a-GI) } \\
\text { DPP-4i: }-2.9 \mathrm{~kg}(10 \mathrm{mg}+\mathrm{DPP}-4 \mathrm{i}) ; \\
-2.8 \mathrm{~kg}(25 \mathrm{mg}+\mathrm{DPP}-4 \mathrm{i}) \\
\text { Glinide: }-2.6 \mathrm{~kg}(10 \mathrm{mg}+\text { glinide); } \\
-3.1 \mathrm{~kg}(25 \mathrm{mg}+\text { glinide })\end{array}$ & - \\
\hline
\end{tabular}


Table 1. Continued

\begin{tabular}{|c|c|c|c|c|}
\hline Study & Design and population & Intervention & Effect on body weight & Effect on waist circumference \\
\hline $\begin{array}{l}\text { Kashiwagi } \\
\text { et al. (2015) } \\
\text { [89] }\end{array}$ & $\begin{array}{l}\text { Phase III, 24-wk ran- } \\
\text { domized study; Japa- } \\
\text { nese T2DM }(n=168)\end{array}$ & $\begin{array}{l}\text { Ipragliflozin (50 mg); } \\
\text { Met; placebo }\end{array}$ & $\begin{array}{l}-2.33 \mathrm{~kg}(50 \mathrm{mg}+\mathrm{Met}) ;-0.63 \mathrm{~kg} \\
\quad(\text { placebo+Met })\end{array}$ & $\begin{array}{l}-2.39 \mathrm{~cm}(50 \mathrm{mg}+\mathrm{Met}) ;-0.48 \mathrm{~cm} \\
\quad(\text { placebo+Met })\end{array}$ \\
\hline $\begin{array}{l}\text { Lu et al. (2016) } \\
\text { [76] }\end{array}$ & $\begin{array}{l}\text { Phase III, 24-wk ran- } \\
\text { domized double- } \\
\text { blind study; Asian } \\
\text { T2DM }(n=171)\end{array}$ & $\begin{array}{l}\text { Ipragliflozin (50 mg); } \\
\text { Met; placebo }\end{array}$ & $\begin{array}{l}-2.93 \mathrm{~kg}(50 \mathrm{mg}+\mathrm{Met}) ;-1.70 \mathrm{~kg} \\
\quad \text { (placebo+Met) }\end{array}$ & $\begin{array}{l}-1.72 \mathrm{~cm}(50 \mathrm{mg}+\mathrm{Met}) ;-0.85 \mathrm{~cm} \\
\quad \text { (placebo+Met) }\end{array}$ \\
\hline $\begin{array}{l}\text { Kashiwagi } \\
\text { et al. (2015) } \\
\text { [90] }\end{array}$ & $\begin{array}{l}\text { Phase III, 24-wk ran- } \\
\text { domized double- } \\
\text { blind study; Japanese } \\
\text { T2DM }(n=152)\end{array}$ & $\begin{array}{l}\text { Ipragliflozin }(50 \mathrm{mg}) \\
\text { pioglitazone; } \\
\text { placebo }\end{array}$ & $\begin{array}{l}-2.29 \mathrm{~kg}(50 \mathrm{mg}+\text { pioglitazone }) ; 0.51 \\
\mathrm{~kg} \text { (placebo+pioglitazone) }\end{array}$ & $\begin{array}{l}-1.82 \mathrm{~cm} \text { (50 mg+pioglitazone); } 0.14 \\
\mathrm{~cm} \text { (placebo+pioglitazone) }\end{array}$ \\
\hline $\begin{array}{l}\text { Kashiwagi } \\
\text { et al. (2015) } \\
\text { [91] }\end{array}$ & $\begin{array}{l}\text { Phase III, 24-wk ran- } \\
\text { domized double- } \\
\text { blind study; Japanese } \\
\text { T2DM }(n=243)\end{array}$ & $\begin{array}{l}\text { Ipragliflozin }(50,100 \\
\text { mg); SU; placebo }\end{array}$ & $\begin{array}{l}-2.33 \mathrm{~kg}(50 \mathrm{mg}+\mathrm{SU}) ;-0.88 \mathrm{~kg} \\
\quad(\text { placebo+SU) }\end{array}$ & $\begin{array}{l}-1.61 \mathrm{~cm}(50 \mathrm{mg}+\mathrm{SU}) ;-0.87 \mathrm{~cm} \\
\quad(\text { placebo+SU) }\end{array}$ \\
\hline $\begin{array}{l}\text { Seino et al. } \\
\text { (2018) [69] }\end{array}$ & $\begin{array}{l}\text { 52-wk, open-label } \\
\text { study; Japanese } \\
\text { T2DM }(n=76)\end{array}$ & $\begin{array}{l}\text { Luseogliflozin ( } 2.5 \\
\text { mg, option to } \\
\text { uptitrate to } 5 \mathrm{mg}) \text {; } \\
\text { liraglutide }\end{array}$ & $\begin{array}{l}12 \mathrm{wk}:-1.65 \mathrm{~kg}(2.5 / 5 \mathrm{mg}+\mathrm{GLP}-\mathrm{RA}) \\
12 \mathrm{wk}-2.52 \mathrm{~kg}(2.5 / 5 \mathrm{mg}+\text { GLP-RA }) \\
36 \mathrm{wk}-2.86 \mathrm{~kg}(2.5 / 5 \mathrm{mg}+\mathrm{GLP}-\mathrm{RA}) \\
52 \mathrm{wk}-2.71 \mathrm{~kg}(2.5 / 5 \mathrm{mg}+\text { GLP-RA })\end{array}$ & $\begin{array}{l}12 \mathrm{wk}:-1.39 \mathrm{~cm}(2.5 / 5 \mathrm{mg}+\mathrm{GLP}-\mathrm{RA}) \\
12 \mathrm{wk}-2.63 \mathrm{~cm}(2.5 / 5 \mathrm{mg}+\mathrm{GLP}-\mathrm{RA}) \\
36 \mathrm{wk}:-3.09 \mathrm{~cm}(2.5 / 5 \mathrm{mg}+\mathrm{GLP}-\mathrm{RA}) \\
52 \mathrm{wk}:-2.86 \mathrm{~cm}(2.5 / 5 \mathrm{mg}+\text { GLP-RA })\end{array}$ \\
\hline $\begin{array}{l}\text { Ikeda et al. } \\
\text { (2015) [92] }\end{array}$ & $\begin{array}{l}\text { Phase II, 12-wk ran- } \\
\text { domized, double- } \\
\text { blind study }(n=398)\end{array}$ & $\begin{array}{l}\text { Tofogliflozin }(2.5,5 \text {, } \\
\text { 10, 20, or } 40 \mathrm{mg}) \\
\text { Met; placebo }\end{array}$ & $\begin{array}{l}-1.56 \mathrm{~kg}(2.5 \mathrm{mg}+\mathrm{Met}) ;-1.85 \mathrm{~kg}(5 \\
\mathrm{mg}+\mathrm{Met}) ;-2.24 \mathrm{~kg}(10 \mathrm{mg}+\mathrm{Met}) ; \\
-2.55 \mathrm{~kg}(20 \mathrm{mg}+\mathrm{Met}) ;-2.82 \mathrm{~kg} \\
\text { (40 mg+Met); }-0.74 \mathrm{~kg} \\
\text { (placebo+Met) }\end{array}$ & - \\
\hline \multicolumn{5}{|l|}{ Add-on to insulin } \\
\hline $\begin{array}{l}\text { Inagaki et al. } \\
(2016)[93]\end{array}$ & $\begin{array}{l}\text { Phase IV, 16-wk dou- } \\
\text { ble-blind random- } \\
\text { ized study; Japanese } \\
\text { T2DM }(n=146)\end{array}$ & $\begin{array}{l}\text { Canagliflozin (100 } \\
\text { mg); insulin; } \\
\text { placebo }\end{array}$ & $\begin{array}{l}-1.49 \mathrm{~kg}(100 \mathrm{mg}+\text { insulin }) ; 0.15 \mathrm{~kg} \\
\quad \text { (placebo+insulin) }\end{array}$ & - \\
\hline $\begin{array}{l}\text { Inagaki et al. } \\
\text { (2018) [94] }\end{array}$ & $\begin{array}{l}\text { Phase IV, 52-wk (16- } \\
\text { wk double-blind } \\
\text { randomized placebo- } \\
\text { controlled study+36- } \\
\text { wk open-label exten- } \\
\text { sion); Japanese } \\
\text { T2DM }(n=146)\end{array}$ & $\begin{array}{l}\text { Canagliflozin (100 } \\
\text { mg); insulin; } \\
\text { placebo }\end{array}$ & $\begin{array}{l}-0.99 \mathrm{~kg} \text { (placebo+insulin } \rightarrow 100 \\
\text { mg+insulin); }-1.52 \mathrm{~kg}(100 \\
\text { mg+insulin } \rightarrow 100 \text { mg+insulin })\end{array}$ & - \\
\hline $\begin{array}{l}\text { Araki et al. } \\
\text { (2016) [70] }\end{array}$ & $\begin{array}{l}\text { Phase IV, 16-wk dou- } \\
\text { ble-blind random- } \\
\text { ized placebo-con- } \\
\text { trolled study; Japa- } \\
\text { nese T2DM }(n=182)\end{array}$ & $\begin{array}{l}\text { Dapagliflozin ( } 5 \mathrm{mg}) \text {; } \\
\text { insulin; placebo }\end{array}$ & $\begin{array}{l}-0.55 \mathrm{~kg}(5 \mathrm{mg}+\text { insulin }) ; 0.66 \mathrm{~kg} \\
\quad \text { (placebo+insulin) }\end{array}$ & $\begin{array}{l}\text { Placebo-corrected mean reduction: } \\
-1.00 \mathrm{~cm}\end{array}$ \\
\hline $\begin{array}{l}\text { Araki et al. } \\
\text { (2017) [71] }\end{array}$ & $\begin{array}{l}\text { 36-wk open-label ex- } \\
\text { tension of Araki et al. } \\
2016[70](n=175)\end{array}$ & $\begin{array}{l}\text { Dapagliflozin (5 mg); } \\
\text { insulin }\end{array}$ & $\begin{array}{l}-1.5 \mathrm{~kg} \text { ( } 5 \mathrm{mg}+\text { insulin } \rightarrow 5 \\
\text { mg+insulin); }-1.37 \mathrm{~kg} \\
\text { (placebo+insulin } \rightarrow 100 \\
\text { mg+insulin) }\end{array}$ & $\begin{array}{l}-1.4 \mathrm{~cm}(5 \mathrm{mg}+\text { insulin } \rightarrow 5 \\
\text { mg+insulin); }-0.9 \mathrm{~cm} \\
\text { (placebo+insulin } \rightarrow 100 \\
\text { mg+insulin) }\end{array}$ \\
\hline $\begin{array}{l}\text { Yang et al. } \\
\text { (2018) [95] }\end{array}$ & $\begin{array}{l}\text { Phase III, 24-wk ran- } \\
\text { domized double- } \\
\text { blind study; Asian } \\
\text { T2DM }(n=272)\end{array}$ & $\begin{array}{l}\text { Dapagliflozin (10 } \\
\text { mg); insulin; } \\
\text { placebo }\end{array}$ & $\begin{array}{l}-1.00 \mathrm{~kg}(10 \mathrm{mg}+\text { insulin }) ; 0.37 \mathrm{~kg} \\
\quad \text { (placebo+insulin) }\end{array}$ & $\begin{array}{l}-0.70 \mathrm{~cm}(10 \mathrm{mg}+\text { insulin }) ; 0.00 \mathrm{~cm} \\
\quad \text { (placebo+insulin) }\end{array}$ \\
\hline
\end{tabular}

(Continued to the next page) 
Table 1. Continued

\begin{tabular}{|c|c|c|c|c|}
\hline Study & Design and population & Intervention & Effect on body weight & Effect on waist circumference \\
\hline $\begin{array}{l}\text { Ishihara et al. } \\
\text { (2016) [96] }\end{array}$ & $\begin{array}{l}\text { Phase IV, 16-wk dou- } \\
\text { ble-blind random- } \\
\text { ized placebo-con- } \\
\text { trolled study; } \\
\text { Japanese T2DM } \\
(n=262)\end{array}$ & $\begin{array}{l}\text { Ipragliflozin }(50 \mathrm{mg}) \\
\text { insulin; placebo }\end{array}$ & $\begin{array}{l}-1.09 \mathrm{~kg}(10 \mathrm{mg}+\text { insulin}) ;-0.05 \mathrm{~kg} \\
\quad \text { (placebo+insulin) }\end{array}$ & $\begin{array}{l}-1.36 \mathrm{~cm}(10 \mathrm{mg}+\text { insulin }) ;-0.84 \\
\mathrm{~cm} \text { (placebo+insulin) }\end{array}$ \\
\hline $\begin{array}{l}\text { Seino et al. } \\
\text { (2018) [77] }\end{array}$ & $\begin{array}{l}\text { Phase IV, 52-wk (16- } \\
\text { wk double-blind } \\
\text { randomized place- } \\
\text { bo-controlled } \\
\text { study+36-wk open- } \\
\text { label extension); } \\
\text { Japanese T2DM } \\
(n=233)\end{array}$ & $\begin{array}{l}\text { Luseogliflozin (2.5 } \\
\text { mg); insulin; } \\
\text { placebo }\end{array}$ & $\begin{array}{l}-1.32 \mathrm{~kg}(2.5 \mathrm{mg}+\text { insulin }) ;-0.05 \\
\text { kg (placebo+insulin) }\end{array}$ & $\begin{array}{l}-1.16 \mathrm{~cm}(2.5 \mathrm{mg}+\text { insulin }) ; 0.04 \\
\mathrm{~cm} \text { (placebo+insulin) }\end{array}$ \\
\hline $\begin{array}{l}\text { Terauchi et al. } \\
\text { (2017) [97] }\end{array}$ & $\begin{array}{l}\text { Phase IV, 16-wk dou- } \\
\text { ble-blind random- } \\
\text { ized placebo-con- } \\
\text { trolled study; } \\
\text { Japanese T2DM } \\
(n=211)\end{array}$ & $\begin{array}{l}\text { Tofogliflozin ( } 20 \mathrm{mg}) \\
\text { insulin; placebo }\end{array}$ & $\begin{array}{l}-1.34 \mathrm{~kg}(20 \mathrm{mg}+\text { insulin }) ; 0.03 \mathrm{~kg} \\
\quad \text { (placebo+insulin) }\end{array}$ & - \\
\hline $\begin{array}{l}\text { Terauchi et al. } \\
\text { (2018) [98] }\end{array}$ & $\begin{array}{l}\text { 36-wk open-label ex- } \\
\text { tension of Terauchi } \\
\text { et al. }(2017) \text { [97] } \\
(n=210)\end{array}$ & $\begin{array}{l}\text { Tofogliflozin }(20 \mathrm{mg}) \\
\text { insulin }\end{array}$ & $\begin{array}{l}-1.52 \mathrm{~kg}(20 \mathrm{mg}+\text { insulin } \rightarrow 20 \\
\text { mg+insulin); }-2.13 \mathrm{~kg} \\
\text { (placebo+insulin } \rightarrow 20 \\
\text { mg+insulin) }\end{array}$ & - \\
\hline $\begin{array}{l}\text { Suzuki et al. } \\
\text { (2016) [99] }\end{array}$ & $\begin{array}{l}\text { 24-wk randomized } \\
\text { active-controlled } \\
\text { study; Japanese } \\
\text { T2DM }(n=53)\end{array}$ & $\begin{array}{l}\text { Tofogliflozin (20 mg); } \\
\text { insulin; insulin } \\
\text { glargine }\end{array}$ & $\begin{array}{c}0.6 \mathrm{~kg} \text { (insulin); }-2.9 \mathrm{~kg}(20 \\
\mathrm{mg}+\text { insulin }) ;-3.4 \mathrm{~kg}(20 \\
\mathrm{mg}+\text { insulin glargine })\end{array}$ & - \\
\hline \multicolumn{5}{|l|}{ Pooled analyses } \\
\hline $\begin{array}{l}\text { John et al. } \\
\qquad(2016)[100]\end{array}$ & $\begin{array}{l}\text { Pooled data from four } \\
26 \text {-wk placebo-con- } \\
\text { trolled studies } \\
(n=2,313) \text { and a } \\
\text { 104-wk active-con- } \\
\text { trolled study } \\
(n=1,450) \text { in T2DM } \\
\text { patients in hot cli- } \\
\text { mate countries }\end{array}$ & $\begin{array}{l}\text { Canagliflozin (100, } \\
300 \text { mg); placebo; } \\
\text { glim+Met; cana- } \\
\text { gliflozin (100, } 300 \\
\text { mg)+Met }\end{array}$ & $\begin{array}{l}\text { Placebo-controlled trials } \\
\text { Hot climate subset: }-1.9 \mathrm{~kg}(100 \\
\text { mg); }-2.4(300 \mathrm{mg}) ;-0.3 \mathrm{~kg} \\
\text { (placebo) } \\
\text { Other climate subset: }-2.8 \mathrm{~kg} \\
\quad(100 \mathrm{mg}) ;-3.4 \mathrm{~kg}(300 \mathrm{mg}) ; \\
\quad-0.7 \mathrm{~kg} \text { (placebo) } \\
\text { Active-controlled trials } \\
\text { Hot climate subset: }-2.6 \mathrm{~kg}(100 \\
\text { mg+Met); -2.8 (300 mg+Met); } \\
\text { +1.3 (glim+Met) } \\
\text { Other climate subset: }-3.9 \mathrm{~kg} \\
\text { (100 mg+Met); }-3.9(300 \\
\text { mg+Met); } 0.6 \text { (glim+Met) }\end{array}$ & - \\
\hline $\begin{array}{l}\text { Yang et al. } \\
\text { (2017) [101] }\end{array}$ & $\begin{array}{l}\text { Pooled data from } \\
\text { eight Phase IIb/III } \\
\text { double-blind place- } \\
\text { bo-controlled trials } \\
\text { of up to } 24 \text { wk } \\
(n=1,453)\end{array}$ & $\begin{array}{l}\text { Dapagliflozin }(5,10 \\
\text { mg); placebo }\end{array}$ & $\begin{array}{l}-1.9 \mathrm{~kg}(5 \mathrm{mg}) ;-2.4 \mathrm{~kg}(10 \mathrm{mg}) ; \\
\quad-0.6 \mathrm{~kg} \text { (placebo })\end{array}$ & - \\
\hline
\end{tabular}


Table 1. Continued

\begin{tabular}{|c|c|c|c|c|}
\hline Study & Design and population & Intervention & Effect on body weight & Effect on waist circumference \\
\hline $\begin{array}{l}\text { Yoon et al. } \\
\text { (2016) [102] }\end{array}$ & $\begin{array}{l}\text { Pooled data from four } \\
\text { Phase III trials up to } \\
24 \mathrm{wk}(n=1,326)\end{array}$ & $\begin{array}{l}\text { Empagliflozin }(10,25 \\
\text { mg); placebo, other } \\
\text { GLDs }\end{array}$ & $\begin{array}{l}\text { Pooled data: }-1.6 \mathrm{~kg}(10 \mathrm{mg}) ;-1.8 \mathrm{~kg} \\
\text { (10 mg) } \\
\text { Monotherapy: }-1.7 \mathrm{~kg}(10 \mathrm{mg}) ; \\
-2.1 \mathrm{~kg}(25 \mathrm{mg}) \\
\text { Met: }-1.4 \mathrm{~kg}(10 \mathrm{mg}+\mathrm{Met}) ;-1.5 \mathrm{~kg} \\
\text { (10 mg+Met) } \\
\text { Met+SU: }-1.5 \mathrm{~kg}(10 \mathrm{mg}+\mathrm{Met}+\mathrm{SU}) ; \\
-1.9 \mathrm{~kg}(10 \mathrm{mg}+\mathrm{Met}+\mathrm{SU}) \\
\text { Pioglitazone: }-2.0 \mathrm{~kg} \\
\text { (10 mg+pioglitazone); } \\
-1.7 \mathrm{~kg}(10 \mathrm{mg}+\text { pioglitazone })\end{array}$ & - \\
\hline $\begin{array}{l}\text { Prasanna } \\
\text { Kumar et al. } \\
\text { (2016) [103] }\end{array}$ & $\begin{array}{l}\text { Pooled subgroup } \\
\text { analysis of patients } \\
\text { enrolled from India } \\
(n=124) \text { in four } \\
\text { randomized Phase } \\
\text { III trials }\end{array}$ & $\begin{array}{l}\text { Canagliflozin (100, } \\
300 \mathrm{mg}) ; \text { placebo; } \\
\text { other GLDs }\end{array}$ & $\begin{array}{l}\text { Overall population: }-2.6(100 \mathrm{mg}) \\
-3.3(300 \mathrm{mg}) \\
\text { Indian subgroup: }-1.7(100 \mathrm{mg}) \\
\quad-2.2(300 \mathrm{mg})\end{array}$ & - \\
\hline $\begin{array}{l}\text { Seino et al. } \\
\text { (2015) [104] }\end{array}$ & $\begin{array}{l}\text { Pooled analysis of two } \\
\text { 52-wk studies; } \\
\text { Japanese T2DM } \\
(n=708)\end{array}$ & $\begin{array}{l}\text { Luseogliflozin (2.5 } \\
\text { mg); other GLDs }\end{array}$ & $\begin{array}{l}\text { SU: }-2.23 \mathrm{~kg}(2.5 \mathrm{mg}+\mathrm{SU}) \\
\text { BG: }-2.86 \mathrm{~kg}(2.5 \mathrm{mg}+\mathrm{BG}) \\
\text { DPP-4i: }-1.96 \mathrm{~kg}(2.5 \mathrm{mg}+\mathrm{DPP}-4 \mathrm{i}) \\
\text { TZD: }-2.32 \mathrm{~kg}(2.5 \mathrm{mg}+\mathrm{TZD}) \\
\text { Glinide: }-2.88 \mathrm{~kg}(2.5 \mathrm{mg}+\mathrm{glinide}) \\
\text { a-GI: }-2.80 \mathrm{~kg}(2.5 \mathrm{mg}+\mathrm{a}-\mathrm{GI}) \\
\text { SU+placebo (24 wk): } 0.16 \mathrm{~kg} \\
\text { (placebo+SU) }\end{array}$ & - \\
\hline \multicolumn{5}{|c|}{ Post-marketing study } \\
\hline $\begin{array}{l}\text { Utsunomiya } \\
\text { et al. }(2017) \\
\text { [105] }\end{array}$ & $\begin{array}{l}\text { 1-yr prospective, ob- } \\
\text { servational, post- } \\
\text { marketing study } \\
(n=1,424)\end{array}$ & Tofogliflozin & $\begin{array}{l}-1.18 \mathrm{~kg}(4 \mathrm{wk}) ;-1.81 \mathrm{~kg}(12 \mathrm{wk}) ; \\
-2.01 \mathrm{~kg}(24 \mathrm{wk}) ;-1.72 \mathrm{~kg}(36 \mathrm{wk}) ; \\
-2.55(52 \mathrm{wk})\end{array}$ & - \\
\hline $\begin{array}{l}\text { Nakamura } \\
\text { et al. }(2018) \\
{[106]}\end{array}$ & $\begin{array}{l}\text { 24-mo post-market- } \\
\text { ing surveillance } \\
\text { study }(n=8,757)\end{array}$ & Ipragliflozin & $\begin{array}{l}-1.32 \mathrm{~kg}(1 \mathrm{mo}) ;-2.16 \mathrm{~kg}(3 \mathrm{mo}) ; \\
-2.45 \mathrm{~kg}(6 \mathrm{mo}) ;-2.81 \mathrm{~kg}(12 \mathrm{mo}) ; \\
-3.11(24 \mathrm{mo})\end{array}$ & - \\
\hline
\end{tabular}

SGLT-2, sodium glucose cotransporter-2; T2DM, type 2 diabetes mellitus; GLD, glucose-lowering drug; SU, sulfonylurea; $\alpha$-GI, alpha glucosidase inhibitor; BG, biguanide; TZD, thiazolidinedione; DPP-4i, dipeptidyl peptidase-4 inhibitor; Met, metformin; GLP-RA, glucagon-like peptide-1 receptor agonist; glim, glimepiride.

DPP-4 inhibitor, on visceral fat reduction in Japanese patients with T2DM. Treatment with ipragliflozin in combination with DPP-4 inhibitor was associated with significant reductions in visceral fat area $(P=0.04)$, subcutaneous fat area $(P=0.004)$, total fat area $(P=0.004)$, and WC $(P=0.001)$, compared with metformin+DPP-4 inhibitor [111].

Furthermore, a 16-week study in Japanese patients with T2DM showed that the decrease in total fat mass accounts for $71 \%$ of total body weight reduction induced by SGLT- 2 inhibitor treatment, with the remainder due to loss in water volume (22\%) [112]. Another study revealed that SGLT-2 inhibitor-in- duced weight loss is not associated with reduction in the muscle mass [113]. In this study, 6-month treatment with dapagliflozin resulted in a significant reduction in body weight and total fat mass, with no significant effect on skeletal muscle mass and psoas muscle index.

\section{Effect on adipose tissue function}

Few studies have assessed the effects of SGLT-2 inhibitors on the adipose tissue in obese Asian patients with T2DM. In a 12week study of dapagliflozin in obese patients with T2DM, there was a significant increase in the plasma adiponectin and 
decrease in high-sensitivity C-reactive protein levels but no significant changes in plasminogen activator inhibitor-1 levels [114].

\section{Effect on ectopic fat}

Obesity results in ectopic fat deposition which leads to impaired insulin sensitivity, non-alcoholic steatohepatitis, impaired insulin secretion, and diastolic dysfunction $[115,116]$. The prospective, open-label, randomized E-LIFT study (effect of empagliflozin on liver fat content in patients with T2DM), examined the effect of empagliflozin $(10 \mathrm{mg} / \mathrm{day}$ as add-on to the standard therapy) on liver fat content (using magnetic resonance imaging-derived proton density fat fraction [MRIPDFF]) in patients with T2DM and NAFLD. At 20 weeks, treatment with empagliflozin significantly reduced liver fat content (mean MRI-PDFF difference: $-4.0 \% ; P<0.0001$ ) and serum alanine aminotransferase levels $(-10.9 \mathrm{IU} / \mathrm{L} ; P=0.005)$, compared with the control group [117]. Another open-label randomized study compared the effects of ipragliflozin and pioglitazone on liver fat content (change in liver-to-spleen [L/S] ratio on computed tomography scan) in patients with T2DM and NAFLD [118]. At 24 weeks, treatment with either ipragliflozin or pioglitazone improved liver fat content, with no significant difference between the two groups (L/S ratio increased by 0.22 in ipragliflozin vs. 0.21 in pioglitazone; $P=$ 0.90). In addition, both treatments reduced the levels of serum aspartate transaminase and alanine transaminase [118].

Furthermore, in two 12-week pilot studies, luseogliflozin and ipragliflozin reduced the epicardial fat volume in overweight/obese patients with T2DM $[119,120]$.

\section{Effect on weight loss-related QoL and treatment satisfaction}

Overweight and obesity negatively affect the patient QoL. A placebo-controlled study in patients with T2DM $(n=180)$ evaluated the effect of dapagliflozin on weight change- and health-related quality of life (HRQoL), using the Study to Help Improve Early evaluation and management of risk factors Leading to Diabetes Weight Questionnaire-9 (SHIELD-WQ9) survey [121]. Treatment with dapagliflozin was associated with improvement in most of the HRQoL domains at week 24, 50 , and 102, and these changes correlated with the weight loss. Similarly, in a study assessing the treatment satisfaction in overweight patients with T2DM ( $n=221)$ using the Oral Hypoglycemic Agent-Questionnaire (OHA-Q) version 2, treat- ment with dapagliflozin was associated with a significant improvement in patient-reported treatment satisfaction [122]. Changes in the OHA-Q total score correlated with changes in body weight and glycosylated hemoglobin. Similar results were observed in a pooled analysis of four randomized controlled trials of canagliflozin [123]. A higher proportion of patients receiving canagliflozin reported interest in continuing treatment upon study completion.

\section{RELATIONSHIP BETWEEN SGLT-2 INHIBITOR-INDUCED WEIGHT LOSS AND IMPROVEMENT OF HYPERGLYCEMIA AND CARDIOVASCULAR OUTCOMES}

Weight loss might contribute to the CV benefits observed with SGLT-2 inhibitors in multiple trials. The randomized Look AHEAD trial studied the impact of weight loss on the primary prevention of $\mathrm{CV}$ outcomes in overweight/obese patients with T2DM [124]. The trial compared the efficacy of intensive lifestyle intervention with diabetes support and education in 5,145 adults with T2DM and a mean BMI of $36.0 \mathrm{~kg} / \mathrm{m}^{2}$. After a median follow-up of 9.6 years, lifestyle intervention resulted in a significant weight loss $(-8.7 \%$ at 1 year, and $-6.0 \%$ at the end of study) but did not show a beneficial effect on mortality and CV outcomes [124]. However, a recent analysis from the study revealed a significant association between magnitude of weight loss and long-term risk of CV outcomes. Participants from both intensive lifestyle intervention and diabetes support and education groups who achieved a weight loss of $>10 \%$ had a significantly lower risk of CV outcomes (composite of CV death, myocardial infarction, stroke, or angina hospitalization; hazard ratio, $0.79 ; 95 \%$ CI, 0.64 to 0.98 ) [125]. In addition, weight loss in the intensive lifestyle intervention group was associated with improved glycemic control, QoL, physical functioning and mobility, and reduced risk of CKD, sleep apnea, urinary incontinence, and depression [126-132]. In other studies, weight loss interventions such as anti-obesity drugs (orlistat, rimonabant, and sibutramine) and bariatric surgery have been shown to improve $\mathrm{CV}$ risk factors (T2DM, hypertension, and hyperlipidemia) in people with obesity [133-135].

The mechanism of SGLT-2 inhibitor-induced weight reduction is likely due to calorie loss and diuresis induced by urinary glucose excretion. A study to quantify the effect of SGLT-2 inhibitor-induced calorie loss on body weight showed a difference in the observed and expected weight loss based on uri- 
nary energy loss [136]. Over 90 weeks, the mean urinary energy loss with empagliflozin treatment was $206 \mathrm{kcal} /$ day, which was expected to result in a weight loss of $-11.3 \mathrm{~kg}$ (assuming no compensatory changes in energy intake), in contrast with the actual weight loss of $-3.2 \mathrm{~kg}$ ( $29 \%$ of the predicted weight loss). This difference in the calorie-to-weight loss was higher in leaner patients (lower BMI) and those with higher eGFR [136]. Considering that the resting and postprandial energy expenditures were unaltered after chronic SGLT-2 inhibitor treatment [137], the difference in the calorie-to-weight loss was attributable to an adaptive increase in calorie intake after 10 weeks of treatment. In addition, the calorie deficit induced by glycosuria leads to a shift in substrate utilization from glucose to lipids, resulting in increased lipolysis and FFA oxidation. Of note, more than two-thirds of SGLT-2 inhibitor-induced weight loss is attributable to a reduction in fat mass $[58,138,139]$. Further- more, recent studies have shown that SLGT-2 is expressed in the pancreatic alpha cells, and its inhibition triggers secretion of glucagon directly. Altered (reduced) insulin-to-glucagon ratio simulates "starvation" state leading to increased lipolysis and mobilization of FFAs $[140,141]$. In addition, studies on diet-induced obese rodent models suggested that SGLT-2 inhibition increases fat utilization (oxidation) and browning of white adipose tissue, reduces hepatic fat content, and attenuates obesity-induced inflammation and insulin resistance [142-144].

The role of weight loss induced by SGLT-2 inhibitors should be considered in combination with their other effects, which include: (1) diuretic and natriuretic effects and reduction in cardiac preload; (2) shift in cardiac energy substrate from fat and glucose oxidation to more efficient ketone bodies; (3) reduced epicardial fat volume leading to a decrease in cardiac fibrosis and enhanced contractility; (4) increase in hematocrit;

Table 2. Clinical recommendations on the use of SGLT-2 inhibitors for the management of Asian patients with T2DM and abdominal obesity

Clinical recommendations

Overweight/obesity burden and associated complications in Asian patients with T2DM

- Overweight/obesity is a major driver of the increasing prevalence of T2DM in Asia, and most of the patients with T2DM are overweight/ obese.

- Asian populations have higher abdominal adiposity for any given BMI compared with their Caucasian counterparts.

- Visceral or abdominal adiposity is strongly associated with risk of adverse CV outcomes and has been shown to be superior to BMI in predicting CVD risk.

- Overweight/obesity in patients with T2DM independently increases the risk of hypertension, dyslipidemia, and CHD; it is also associated with other complications such as ectopic fat deposition (e.g., NAFLD).

Weight loss as an essential management goal in overweight/obese patients with T2DM

- Weight loss is an important management goal in patients with T2DM, who are overweight/obese.

- Lifestyle modification (diet, physical activity, and behavioral therapy) designed to achieve and maintain weight loss should be prescribed for patients with T2DM who are overweight/obese.

- Limited glucose-lowering pharmacological therapies are available that promote weight loss in T2DM.

- In overweight/obese patients with T2DM, use of glucose-lowering medications that promote weight loss or are weight neutral is recommended.

- Use of medications that are associated with weight gain should be minimized in overweight/obese patients with T2DM.

Role of SGLT-2 inhibitors in the management of Asian patients with T2DM and abdominal obesity

- SGLT-2 inhibitor is recommended to promote weight loss and reduction in visceral adiposity in Asian patients with T2DM and abdominal obesity.

- SGLT-2 inhibitors promote weight loss in Asian patients with T2DM as monotherapy or in combination with other GLDs. - More than two-thirds of SGLT-2 inhibitor-induced weight loss is attributable to a reduction in body fat mass.

- SGLT-2 inhibitor treatment reduces WC (a measure of abdominal or visceral adiposity) and ectopic fat deposition in non-adipose tissues, and improves adipose tissue function, HRQoL, and patient-reported treatment satisfaction.

SGLT-2, sodium glucose cotransporter-2; T2DM, type 2 diabetes mellitus; BMI, body mass index; CV, cardiovascular; CVD, cardiovascular disease; CHD, coronary heart disease; NAFLD, non-alcoholic fatty liver disease; GLD, glucose-lowering drug; WC, waist circumference; HRQoL, health-related quality of life. 
and (5) renoprotective effects [58,139,145,146]. In addition, SGLT-2 inhibitors have been shown to enhance utilization of FFAs and improve adipose tissue function. These effects may lead to a reduction in inflammation, improvements in insulin resistance, glycemic control, and blood pressure control.

\section{CONCLUSIONS}

Management of overweight/obese patients with T2DM poses a challenge to clinicians because of there being fewer therapeutic options available for both glycemic control and weight reduction. Some of the older, established GLDs induce weight gain as a side effect. SGLT-2 inhibitors are novel oral GLDs that promote weight loss, decrease adiposity, and improve CV outcomes. As most of the T2DM patients are overweight/obese and at high risk of CVD, SGLT-2 inhibitors offer an effective therapeutic option for the management of these patients, particularly in the Asian region, which has a high burden of abdominal adiposity and CVD. Based on the current evidence on SGLT-2 inhibitors in the Asian population, results from the large CVOTs, and clinical experience, a series of clinical recommendations have been developed (Table 2).

\section{CONFLICTS OF INTEREST}

The development of these expert recommendations was supported by an unrestricted educational grant from AstraZeneca Ltd, who had no influence on the content. All authors have read, approved, and take full responsibility for the accuracy of the content.

Wayne Huey-Herng Sheu has been advisor and/or speaker for AstraZeneca, Bayer HealthCare, Boehringer Ingelheim Pharmaceuticals, Daiichi-Sankyo, Eli Lilly and Company, Merck Sharp \& Dohme, Mitsubishi Tanabe Pharma Corporation, Novartis Pharmaceuticals, Novo Nordisk, Pfizer, Sanofi, and Takeda Pharmaceutical Company.

Chan Siew Pheng has received honoraria as speaker and advisor for AstraZeneca, Boehringer Ingelheim, Eli Lilly, Janssen, Merck, Merck Sharp \& Dohme, Novartis, Novo Nordisk, Sanofi, and Servier.

Bien J. Matawaran has received honoraria and CME grants from AstraZeneca, Boehringer Ingelheim, Eli Lilly, Merck, Multicare, Merck Sharp \& Dohme, NatraPharm, Novartis, Novo Nordisk, Pfizer, Sanofi, Servier, and Torrent Pharma.

Chaicharn Deerochanawong has received honoraria as the speaker or advisor or research grants from AstraZeneca, Boehringer Ingelheim, Janssen, Bayer, Eli Lilly, Abbott, Novartis, Pfizer, Merck Sharp \& Dohme, Novo Nordisk, Sanofi, and Takeda.

Ambrish Mithal has received honoraria as speaker and advisor from AstraZeneca, Abbott, Boehringer Ingelheim, Cipla, Dr. Reddy's, Eli Lilly, Glenmark, GlaxoSmithKline, Ipca Laboratories, Janssen, Lupin, Merck Sharp \& Dohme, Novartis, Novo Nordisk, Pfizer, Sanofi, Serdia Servier, Sun Pharma, Torrent, Wockhardt and Zydus Nutrition.

Juliana Chan is the Chief Executive Officer (on probono basis) of Asia Diabetes Foundation, a charitable foundation established under The Chinese University of Hong Kong Foundation for developing the JADE Technology. She has received honoraria and traveling support for consultancy or giving lectures and her affiliated institutions have received research and educational grants from Amgen, Ascencia, AstraZeneca, Bayer, Bristol-Myers Squibb, Boehringer Ingelheim, Daiichi-Sankyo, Eli Lilly, GlaxoSmithKline, Medtronic, Merck Serono, Merck Sharp \& Dohme, Novo Nordisk, Pfizer, and Sanofi. Ketut Suastika has received honoraria as a speaker or advisor from AstraZeneca, Boehringer Ingelheim, Merck Sharp \& Dohme, Novo Nordisk, Sanofi, Merck, and Servier.

Chin Meng Khoo has received honoraria as a speaker or advisor from AstraZeneca, Boehringer Ingelheim, Novo Nordisk, and Sanofi.

Nguyen Huu Man has no disclosures.

Ji Linong is a member of the DISCOVER Scientific Committee and received support from AstraZeneca to attend DISCOVER planning and update meetings. He has also received honoraria from Eli Lilly, Bristol-Myers Squibb, Novartis, Novo Nordisk, Bayer, Merck Sharp \& Dohme, Takeda, Sanofi, Roche, Boehringer Ingelheim, and AstraZeneca; research support from Roche, Sanofi, Merck Sharp \& Dohme, AstraZeneca, Novartis, Eli Lilly, and Bristol-Myers Squibb.

Andrea Luk is a member of advisory boards for AstraZeneca, Boehringer Ingelheim, Sanofi, and Amgen. She has received research grants from Boehringer Ingelheim, Merck Sharp \& Dohme, Sanofi, and Amgen; and travel grants from AstraZeneca, Boehringer Ingelheim, Merck Sharp \& Dohme, Novartis, Novo Nordisk, and Sanofi.

Kun-Ho Yoon has received honoraria as a speaker or advisor from AstraZeneca, Boehringer Ingelheim, Eli Lilly, Hanmi Pharmaceutical, Merck Sharp \& Dohme, Novo Nordisk, Sanofi, and Takeda; research support from AstraZeneca and Takeda. 
Medical writing assistance was provided by Syed Abdul Haseeb (MS, CMPP) of MediTech Media, Asia Pacific, and was funded by AstraZeneca Ltd.

\section{ORCID}

Wayne Huey-Herng Sheu https://orcid.org/0000-0002-8805-8340 Kun-Ho Yoon https://orcid.org/0000-0002-9109-2208

\section{ACKNOWLEDGMENTS}

None

\section{REFERENCES}

1. Scheen AJ, Van Gaal LF. Combating the dual burden: therapeutic targeting of common pathways in obesity and type 2 diabetes. Lancet Diabetes Endocrinol 2014;2:911-22.

2. Eckel RH, Kahn SE, Ferrannini E, Goldfine AB, Nathan DM, Schwartz MW, Smith RJ, Smith SR. Obesity and type 2 diabetes: what can be unified and what needs to be individualized? J Clin Endocrinol Metab 2011;96:1654-63.

3. Sears B, Perry M. The role of fatty acids in insulin resistance. Lipids Health Dis 2015;14:121.

4. Bodenant M, Kuulasmaa K, Wagner A, Kee F, Palmieri L, Ferrario MM, Montaye M, Amouyel P, Dallongeville J; MORGAM Project. Measures of abdominal adiposity and the risk of stroke: the MOnica Risk, Genetics, Archiving and Monograph (MORGAM) study. Stroke 2011;42:2872-7.

5. Casanueva FF, Moreno B, Rodriguez-Azeredo R, Massien C, Conthe P, Formiguera X, Barrios V, Balkau B. Relationship of abdominal obesity with cardiovascular disease, diabetes and hyperlipidaemia in Spain. Clin Endocrinol (Oxf) 2010;73:3540.

6. Balkau B, Deanfield JE, Despres JP, Bassand JP, Fox KA, Smith SC Jr, Barter P, Tan CE, van Gaal L, Wittchen HU, Massien C, Haffner SM. International Day for the Evaluation of Abdominal Obesity (IDEA): a study of waist circumference, cardiovascular disease, and diabetes mellitus in 168,000 primary care patients in 63 countries. Circulation 2007;116:1942-51.

7. Lim U, Ernst T, Buchthal SD, Latch M, Albright CL, Wilkens LR, Kolonel LN, Murphy SP, Chang L, Novotny R, Le Marchand $\mathrm{L}$. Asian women have greater abdominal and visceral adiposity than Caucasian women with similar body mass index. Nutr Diabetes 2011;1:e6.
8. Sniderman AD, Bhopal R, Prabhakaran D, Sarrafzadegan N, Tchernof A. Why might South Asians be so susceptible to central obesity and its atherogenic consequences?: the adipose tissue overflow hypothesis. Int J Epidemiol 2007;36:220-5.

9. Wells JC. Commentary: why are South Asians susceptible to central obesity?: the El Nino hypothesis. Int J Epidemiol 2007; 36:226-7.

10. WHO Expert Consultation. Appropriate body-mass index for Asian populations and its implications for policy and intervention strategies. Lancet 2004;363:157-63.

11. World Health Organization. Waist circumference and waisthip ratio: report of a WHO expert consultation, Geneva, 8-11 December 2008. Geneva: World Health Organization; 2011.

12. International Diabetes Federation: The IDF consensus worldwide definition of the metabolic syndrome. Available from: https://www.idf.org/component/attachments/attachments. html?id=705\&task=download (cited 2020 Jan 28).

13. Lee SY, Park HS, Kim DJ, Han JH, Kim SM, Cho GJ, Kim DY, Kwon HS, Kim SR, Lee CB, Oh SJ, Park CY, Yoo HJ. Appropriate waist circumference cutoff points for central obesity in Korean adults. Diabetes Res Clin Pract 2007;75:72-80.

14. American Diabetes Association. Standards of medical care in diabetes: 2019. Diabetes Care 2019;42(Suppl 1):S1-193.

15. Garber AJ, Abrahamson MJ, Barzilay JI, Blonde L, Bloomgarden ZT, Bush MA, Dagogo-Jack S, DeFronzo RA, Einhorn D, Fonseca VA, Garber JR, Garvey WT, Grunberger G, Handelsman Y, Hirsch IB, Jellinger PS, McGill JB, Mechanick JI, Rosenblit PD, Umpierrez GE. Consensus statement by the American Association of Clinical Endocrinologists and American College of Endocrinology on the comprehensive type 2 diabetes management algorithm: 2018 executive summary. Endocr Pract 2018;24:91-120.

16. Deerochanawong C, Chan SP, Matawaran BJ, Sheu WH, Chan J, Man NH, Suastika K, Khoo CM, Yoon KH, Luk A, Mithal A, Linong J. Use of sodium-glucose co-transporter-2 inhibitors in patients with type 2 diabetes mellitus and multiple cardiovascular risk factors: an Asian perspective and expert recommendations. Diabetes Obes Metab 2019;21:2354-67.

17. Ng M, Fleming T, Robinson M, Thomson B, Graetz N, Margono C, Mullany EC, Biryukov S, Abbafati C, Abera SF, Abraham JP, Abu-Rmeileh NM, Achoki T, AlBuhairan FS, Alemu ZA, Alfonso R, Ali MK, Ali R, Guzman NA, Ammar W, Anwari P, Banerjee A, Barquera S, Basu S, Bennett DA, Bhutta Z, Blore J, Cabral N, Nonato IC, Chang JC, Chowdhury R, Courville KJ, Criqui MH, Cundiff DK, Dabhadkar KC, Dandona L, 
Davis A, Dayama A, Dharmaratne SD, Ding EL, Durrani AM, Esteghamati A, Farzadfar F, Fay DF, Feigin VL, Flaxman A, Forouzanfar MH, Goto A, Green MA, Gupta R, Hafezi-Nejad N, Hankey GJ, Harewood HC, Havmoeller R, Hay S, Hernandez L, Husseini A, Idrisov BT, Ikeda N, Islami F, Jahangir E, Jassal SK, Jee SH, Jeffreys M, Jonas JB, Kabagambe EK, Khalifa SE, Kengne AP, Khader YS, Khang YH, Kim D, Kimokoti RW, Kinge JM, Kokubo Y, Kosen S, Kwan G, Lai T, Leinsalu M, Li Y, Liang X, Liu S, Logroscino G, Lotufo PA, Lu Y, Ma J, Mainoo NK, Mensah GA, Merriman TR, Mokdad AH, Moschandreas J, Naghavi M, Naheed A, Nand D, Narayan KM, Nelson EL, Neuhouser ML, Nisar MI, Ohkubo T, Oti SO, Pedroza A, Prabhakaran D, Roy N, Sampson U, Seo H, Sepanlou SG, Shibuya K, Shiri R, Shiue I, Singh GM, Singh JA, Skirbekk V, Stapelberg NJ, Sturua L, Sykes BL, Tobias M, Tran BX, Trasande L, Toyoshima H, van de Vijver S, Vasankari TJ, Veerman JL, Velasquez-Melendez G, Vlassov VV, Vollset SE, Vos T, Wang C, Wang X, Weiderpass E, Werdecker A, Wright JL, Yang YC, Yatsuya H, Yoon J, Yoon SJ, Zhao Y, Zhou M, Zhu S, Lopez AD, Murray CJ, Gakidou E. Global, regional, and national prevalence of overweight and obesity in children and adults during 1980-2013: a systematic analysis for the Global Burden of Disease Study 2013. Lancet 2014;384:766-81.

18. World Health Orgaization: Obesity and overweight. Available from: http://www.who.int/en/news-room/fact-sheets/detail/ obesity-and-overweight (updated 2018 Feb 16).

19. GBD 2015 Obesity Collaborators, Afshin A, Forouzanfar MH, Reitsma MB, Sur P, Estep K, Lee A, Marczak L, Mokdad AH, Moradi-Lakeh M, Naghavi M, Salama JS, Vos T, Abate KH, Abbafati C, Ahmed MB, Al-Aly Z, Alkerwi A, Al-Raddadi R, Amare AT, Amberbir A, Amegah AK, Amini E, Amrock SM, Anjana RM, Arnlov J, Asayesh H, Banerjee A, Barac A, Baye E, Bennett DA, Beyene AS, Biadgilign S, Biryukov S, Bjertness E, Boneya DJ, Campos-Nonato I, Carrero JJ, Cecilio P, Cercy K, Ciobanu LG, Cornaby L, Damtew SA, Dandona L, Dandona R, Dharmaratne SD, Duncan BB, Eshrati B, Esteghamati A, Feigin VL, Fernandes JC, Furst T, Gebrehiwot TT, Gold A, Gona PN, Goto A, Habtewold TD, Hadush KT, Hafezi-Nejad N, Hay SI, Horino M, Islami F, Kamal R, Kasaeian A, Katikireddi SV, Kengne AP, Kesavachandran CN, Khader YS, Khang YH, Khubchandani J, Kim D, Kim YJ, Kinfu Y, Kosen S, Ku T, Defo BK, Kumar GA, Larson HJ, Leinsalu M, Liang X, Lim SS, Liu P, Lopez AD, Lozano R, Majeed A, Malekzadeh R, Malta DC, Mazidi M, McAlinden C, McGarvey ST, Mengistu DT, Mensah GA, Mensink GBM, Mezgebe HB, Mirrakhimov
EM, Mueller UO, Noubiap JJ, Obermeyer CM, Ogbo FA, Owolabi MO, Patton GC, Pourmalek F, Qorbani M, Rafay A, Rai RK, Ranabhat CL, Reinig N, Safiri S, Salomon JA, Sanabria JR, Santos IS, Sartorius B, Sawhney M, Schmidhuber J, Schutte AE, Schmidt MI, Sepanlou SG, Shamsizadeh M, Sheikhbahaei S, Shin MJ, Shiri R, Shiue I, Roba HS, Silva DAS, Silverberg JI, Singh JA, Stranges S, Swaminathan S, Tabares-Seisdedos R, Tadese F, Tedla BA, Tegegne BS, Terkawi AS, Thakur JS, Tonelli M, Topor-Madry R, Tyrovolas S, Ukwaja KN, Uthman OA, Vaezghasemi M, Vasankari T, Vlassov VV, Vollset SE, Weiderpass E, Werdecker A, Wesana J, Westerman R, Yano Y, Yonemoto N, Yonga G, Zaidi Z, Zenebe ZM, Zipkin B, Murray CJL. Health effects of overweight and obesity in 195 countries over 25 years. N Engl J Med 2017;377:13-27.

20. Helble M, Francisco K. The imminent obesity crisis in Asia and the Pacific: first cost estimates. Tokyo: Asian Development Bank Institute; 2017.

21. International Diabetes Federation: IDF diabetes atlas 8th edition. Available from: https://www.idf.org/component/attachments/attachments.html?id=1405\&task=download (cited 2020 Jan 28).

22. Daousi C, Casson IF, Gill GV, MacFarlane IA, Wilding JP, Pinkney JH. Prevalence of obesity in type 2 diabetes in secondary care: association with cardiovascular risk factors. Postgrad Med J 2006;82:280-4.

23. So WY, Raboca J, Sobrepena L, Yoon KH, Deerochanawong C, Ho LT, Himathongkam T, Tong P, Lyubomirsky G, Ko G, Nan H, Chan J; JADE Program Research Team. Comprehensive risk assessments of diabetic patients from seven Asian countries: the Joint Asia Diabetes Evaluation (JADE) program. J Diabetes 2011;3:109-18.

24. Kim BY, Won JC, Lee JH, Kim HS, Park JH, Ha KH, Won KC, Kim DJ, Park KS. Diabetes fact sheets in Korea, 2018: an appraisal of current status. Diabetes Metab J 2019;43:487-94.

25. Ford ES. Body mass index, diabetes, and C-reactive protein among U.S. adults. Diabetes Care 1999;22:1971-7.

26. Ridker PM, Rifai N, Rose L, Buring JE, Cook NR. Comparison of C-reactive protein and low-density lipoprotein cholesterol levels in the prediction of first cardiovascular events. $\mathrm{N}$ Engl J Med 2002;347:1557-65.

27. van Dijk EJ, Prins ND, Vermeer SE, Vrooman HA, Hofman A, Koudstaal PJ, Breteler MM. C-reactive protein and cerebral small-vessel disease: the Rotterdam Scan Study. Circulation 2005;112:900-5.

28. Ni Mhurchu C, Rodgers A, Pan WH, Gu DF, Woodward M; 
Asia Pacific Cohort Studies Collaboration. Body mass index and cardiovascular disease in the Asia-Pacific Region: an overview of 33 cohorts involving 310000 participants. Int J Epidemiol 2004;33:751-8.

29. Asia Pacific Cohort Studies Collaboration. Central obesity and risk of cardiovascular disease in the Asia Pacific Region. Asia Pac J Clin Nutr 2006;15:287-92.

30. Iso H, Sato S, Kitamura A, Imano H, Kiyama M, Yamagishi K, Cui R, Tanigawa T, Shimamoto T. Metabolic syndrome and the risk of ischemic heart disease and stroke among Japanese men and women. Stroke 2007;38:1744-51.

31. Huxley RR, Barzi F, Woo J, Giles G, Lam TH, Rahimi K, Konety S, Ohkubo T, Jee SH, Fang X, Woodward M; Asia Pacific Cohort Studies Collaboration. A comparison of risk factors for mortality from heart failure in Asian and non-Asian populations: an overview of individual participant data from 32 prospective cohorts from the Asia-Pacific Region. BMC Cardiovasc Disord 2014;14:61.

32. Ahmad FS, Ning H, Rich JD, Yancy CW, Lloyd-Jones DM, Wilkins JT. Hypertension, obesity, diabetes, and heart failurefree survival: the cardiovascular disease lifetime risk pooling project. JACC Heart Fail 2016;4:911-9.

33. Maric-Bilkan C. Obesity and diabetic kidney disease. Med Clin North Am 2013;97:59-74.

34. Pinto-Sietsma SJ, Navis G, Janssen WM, de Zeeuw D, Gans RO, de Jong PE; PREVEND Study Group. A central body fat distribution is related to renal function impairment, even in lean subjects. Am J Kidney Dis 2003;41:733-41.

35. Thoenes M, Reil JC, Khan BV, Bramlage P, Volpe M, Kirch W, Bohm M. Abdominal obesity is associated with microalbuminuria and an elevated cardiovascular risk profile in patients with hypertension. Vasc Health Risk Manag 2009;5:577-85.

36. Hill CJ, Cardwell CR, Maxwell AP, Young RJ, Matthews B, O'Donoghue DJ, Fogarty DG. Obesity and kidney disease in type 1 and 2 diabetes: an analysis of the National Diabetes $\mathrm{Au}$ dit. QJM 2013;106:933-42.

37. Thomas G, Sehgal AR, Kashyap SR, Srinivas TR, Kirwan JP, Navaneethan SD. Metabolic syndrome and kidney disease: a systematic review and meta-analysis. Clin J Am Soc Nephrol 2011;6:2364-73.

38. Smith BW, Adams LA. Nonalcoholic fatty liver disease and diabetes mellitus: pathogenesis and treatment. Nat Rev Endocrinol 2011;7:456-65.

39. Pearson-Stuttard J, Zhou B, Kontis V, Bentham J, Gunter MJ, Ezzati M. Worldwide burden of cancer attributable to diabetes and high body-mass index: a comparative risk assessment. Lancet Diabetes Endocrinol 2018;6:e6-15.

40. Bonsignore MR, Borel AL, Machan E, Grunstein R. Sleep apnoea and metabolic dysfunction. Eur Respir Rev 2013;22:35364.

41. The Diabetes Association of the Republic of China: DAROC Clinical Practice Guidelines for diabetes care 2018, Taiwan. Available from: http://www.endo-dm.org.tw/dia/direct/index. asp?BK_KIND $=29 \&$ current $=2018 \% \mathrm{E} 7 \% \mathrm{~B} 3 \% 96 \% \mathrm{E} \% \mathrm{~B} 0 \% \mathrm{~B}$ F\%E7\%97\%85\%E8\%87\%A8\%E5\%BA\%8A\%E7\%85\%A7\%E8 \%AD\%B7\%E6\%8C\%87\%E5\%BC\%95+++++++++++++++++ ++ (cited 2020 Jan 28).

42. Health Technology Assessment Section, Medical Development Division, Ministry of Health Malaysia: Clinical practice guidelines: management of type 2 diabetes mellitus. Available from: http://www.acadmed.org.my/view_file.cfm?fileid=763 (cited 2020 Jan 28).

43. Weng J, Ji L, Jia W, Lu J, Zhou Z, Zou D, Zhu D, Chen L, Chen L, Guo L, Guo X, Ji Q, Li Q, Li X, Liu J, Ran X, Shan Z, Shi L, Song G, Yang L, Yang Y, Yang W; Chinese Diabetes Society. Standards of care for type 2 diabetes in China. Diabetes Metab Res Rev 2016;32:442-58.

44. Ministry of Health Singapore: Diabetes mellitus: $\mathrm{MOH}$ clinical practice guidelines. Available from: https://www.moh.gov. sg/docs/librariesprovider4/guidelines/cpg_diabetes-mellitusbooklet---jul-2014.pdf (cited 2020 Jan 28).

45. Kim MK, Ko SH, Kim BY, Kang ES, Noh J, Kim SK, Park SO, Hur KY, Chon S, Moon MK, Kim NH, Kim SY, Rhee SY, Lee KW, Kim JH, Rhee EJ, Chun S, Yu SH, Kim DJ, Kwon HS, Park KS; Committee of Clinical Practice Guidelines, Korean Diabetes Association. 2019 Clinical practice guidelines for type 2 diabetes mellitus in Korea. Diabetes Metab J 2019;43: 398-406.

46. Garvey WT, Mechanick JI, Brett EM, Garber AJ, Hurley DL, Jastreboff AM, Nadolsky K, Pessah-Pollack R, Plodkowski R; Reviewers of the AACE/ACE Obesity Clinical Practice Guidelines. American Association of Clinical Endocrinologists and American College of endocrinology comprehensive clinical practice guidelines for medical care of patients with obesity. Endocr Pract 2016;22 Suppl 3:1-203.

47. Jensen MD, Ryan DH, Apovian CM, Ard JD, Comuzzie AG, Donato KA, Hu FB, Hubbard VS, Jakicic JM, Kushner RF, Loria $\mathrm{CM}$, Millen BE, Nonas CA, Pi-Sunyer FX, Stevens J, Stevens VJ, Wadden TA, Wolfe BM, Yanovski SZ; American College of Cardiology/American Heart Association Task Force on 
Practice Guidelines; Obesity Society. 2013 AHA/ACC/TOS guideline for the management of overweight and obesity in adults: a report of the American College of Cardiology/American Heart Association Task Force on Practice Guidelines and The Obesity Society. J Am Coll Cardiol 2014;63(25 Pt B): 2985-3023.

48. Inzucchi SE, Bergenstal RM, Buse JB, Diamant M, Ferrannini E, Nauck M, Peters AL, Tsapas A, Wender R, Matthews DR. Management of hyperglycaemia in type 2 diabetes, 2015: a patient-centred approach: update to a position statement of the American Diabetes Association and the European Association for the Study of Diabetes. Diabetologia 2015;58:429-42.

49. Rosenstock J, Hassman DR, Madder RD, Brazinsky SA, Farrell J, Khutoryansky N, Hale PM; Repaglinide Versus Nateglinide Comparison Study Group. Repaglinide versus nateglinide monotherapy: a randomized, multicenter study. Diabetes Care 2004;27:1265-70.

50. Schernthaner G, Grimaldi A, Di Mario U, Drzewoski J, Kempler P, Kvapil M, Novials A, Rottiers R, Rutten GE, Shaw KM. GUIDE study: double-blind comparison of once-daily gliclazide MR and glimepiride in type 2 diabetic patients. Eur J Clin Invest 2004;34:535-42.

51. Dormandy JA, Charbonnel B, Eckland DJ, Erdmann E, MassiBenedetti M, Moules IK, Skene AM, Tan MH, Lefebvre PJ, Murray GD, Standl E, Wilcox RG, Wilhelmsen L, Betteridge J, Birkeland K, Golay A, Heine RJ, Koranyi L, Laakso M, Mokan M, Norkus A, Pirags V, Podar T, Scheen A, Scherbaum W, Schernthaner G, Schmitz O, Skrha J, Smith U, Taton J; PROactive Investigators. Secondary prevention of macrovascular events in patients with type 2 diabetes in the PROactive Study (PROspective pioglitAzone Clinical Trial In macroVascular Events): a randomized controlled trial. Lancet 2005;366:127989.

52. Kahn SE, Haffner SM, Heise MA, Herman WH, Holman RR, Jones NP, Kravitz BG, Lachin JM, O’Neill MC, Zinman B, Viberti G; ADOPT Study Group. Glycemic durability of rosiglitazone, metformin, or glyburide monotherapy. N Engl J Med 2006;355:2427-43.

53. Bolen S, Feldman L, Vassy J, Wilson L, Yeh HC, Marinopoulos S, Wiley C, Selvin E, Wilson R, Bass EB, Brancati FL. Systematic review: comparative effectiveness and safety of oral medications for type 2 diabetes mellitus. Ann Intern Med 2007;147: 386-99.

54. Phung OJ, Scholle JM, Talwar M, Coleman CI. Effect of noninsulin antidiabetic drugs added to metformin therapy on gly- cemic control, weight gain, and hypoglycemia in type 2 diabetes. JAMA 2010;303:1410-8.

55. Karagiannis T, Paschos P, Paletas K, Matthews DR, Tsapas A. Dipeptidyl peptidase- 4 inhibitors for treatment of type 2 diabetes mellitus in the clinical setting: systematic review and meta-analysis. BMJ 2012;344:e1369.

56. Saenz A, Fernandez-Esteban I, Mataix A, Ausejo M, Roque M, Moher D. Metformin monotherapy for type 2 diabetes mellitus. Cochrane Database Syst Rev 2005;3:CD002966.

57. Davies MJ, D’Alessio DA, Fradkin J, Kernan WN, Mathieu C, Mingrone G, Rossing P, Tsapas A, Wexler DJ, Buse JB. Management of hyperglycaemia in type 2 diabetes, 2018: a consensus report by the American Diabetes Association (ADA) and the European Association for the Study of Diabetes (EASD). Diabetologia 2018;61:2461-98.

58. Heerspink HJ, Perkins BA, Fitchett DH, Husain M, Cherney DZ. Sodium glucose cotransporter 2 inhibitors in the treatment of diabetes mellitus: cardiovascular and kidney effects, potential mechanisms, and clinical applications. Circulation 2016;134:752-72.

59. Neal B, Perkovic V, Mahaffey KW, de Zeeuw D, Fulcher G, Erondu N, Shaw W, Law G, Desai M, Matthews DR; CANVAS Program Collaborative Group. Canagliflozin and cardiovascular and renal events in type 2 diabetes. N Engl J Med 2017;377:644-57.

60. Wiviott SD, Raz I, Bonaca MP, Mosenzon O, Kato ET, Cahn A, Silverman MG, Zelniker TA, Kuder JF, Murphy SA, Bhatt DL, Leiter LA, McGuire DK, Wilding JPH, Ruff CT, Gause-Nilsson IAM, Fredriksson M, Johansson PA, Langkilde AM, Sabatine MS; DECLARE-TIMI 58 Investigators. Dapagliflozin and cardiovascular outcomes in type 2 diabetes. $\mathrm{N}$ Engl J Med 2019;380:347-57.

61. Zinman B, Wanner C, Lachin JM, Fitchett D, Bluhmki E, Hantel S, Mattheus M, Devins T, Johansen OE, Woerle HJ, Broedl UC, Inzucchi SE; EMPA-REG OUTCOME Investigators. Empagliflozin, cardiovascular outcomes, and mortality in type 2 diabetes. N Engl J Med 2015;373:2117-28.

62. Cai X, Yang W, Gao X, Chen Y, Zhou L, Zhang S, Han X, Ji L. The association between the dosage of SGLT2 inhibitor and weight reduction in type 2 diabetes patients: a meta-analysis. Obesity (Silver Spring) 2018;26:70-80.

63. Gill HK, Kaur P, Mahendru S, Mithal A. Adverse effect profile and effectiveness of sodium glucose co-transporter 2 inhibitors (SGLT2i): a prospective real-world setting study. Indian J Endocrinol Metab 2019;23:50-5. 
64. Vasilakou D, Karagiannis T, Athanasiadou E, Mainou M, Liakos A, Bekiari E, Sarigianni M, Matthews DR, Tsapas A. Sodium-glucose cotransporter 2 inhibitors for type 2 diabetes: a systematic review and meta-analysis. Ann Intern Med 2013; 159:262-74.

65. Kaku K, Watada H, Iwamoto Y, Utsunomiya K, Terauchi Y, Tobe K, Tanizawa Y, Araki E, Ueda M, Suganami H, Watanabe D; Tofogliflozin 003 Study Group. Efficacy and safety of monotherapy with the novel sodium/glucose cotransporter-2 inhibitor tofogliflozin in Japanese patients with type 2 diabetes mellitus: a combined Phase 2 and 3 randomized, placebo-controlled, double-blind, parallel-group comparative study. Cardiovasc Diabetol 2014;13:65.

66. Kashiwagi A, Kazuta K, Yoshida S, Nagase I. Randomized, placebo-controlled, double-blind glycemic control trial of novel sodium-dependent glucose cotransporter 2 inhibitor ipragliflozin in Japanese patients with type 2 diabetes mellitus. J Diabetes Investig 2014;5:382-91.

67. Seino Y, Kaku K, Inagaki N, Haneda M, Sasaki T, Fukatsu A, Ubukata M, Sakai S, Samukawa Y. Fifty-two-week long-term clinical study of luseogliflozin as monotherapy in Japanese patients with type 2 diabetes mellitus inadequately controlled with diet and exercise. Endocr J 2015;62:593-603.

68. Harashima SI, Inagaki N, Kondo K, Maruyama N, Otsuka M, Kawaguchi Y, Watanabe Y. Efficacy and safety of canagliflozin as add-on therapy to a glucagon-like peptide-1 receptor agonist in Japanese patients with type 2 diabetes mellitus: a 52week, open-label, phase IV study. Diabetes Obes Metab 2018; 20:1770-5.

69. Seino Y, Yabe D, Sasaki T, Fukatsu A, Imazeki H, Ochiai H, Sakai S. Sodium-glucose cotransporter-2 inhibitor luseogliflozin added to glucagon-like peptide 1 receptor agonist liraglutide improves glycemic control with bodyweight and fat mass reductions in Japanese patients with type 2 diabetes: a 52-week, open-label, single-arm study. J Diabetes Investig 2018;9:332-40.

70. Araki E, Onishi Y, Asano M, Kim H, Ekholm E, Johnsson E, Yajima T. Efficacy and safety of dapagliflozin in addition to insulin therapy in Japanese patients with type 2 diabetes: results of the interim analysis of 16-week double-blind treatment period. J Diabetes Investig 2016;7:555-64.

71. Araki E, Onishi Y, Asano M, Kim H, Yajima T. Efficacy and safety of dapagliflozin over 1 year as add-on to insulin therapy in Japanese patients with type 2 diabetes: the DAISY (Dapagliflozin Added to patients under InSulin therapY) trial. Dia- betes Obes Metab 2017;19:562-70.

72. Inagaki N, Kondo K, Yoshinari T, Kuki H. Efficacy and safety of canagliflozin alone or as add-on to other oral antihyperglycemic drugs in Japanese patients with type 2 diabetes: a 52week open-label study. J Diabetes Investig 2015;6:210-8.

73. Inagaki N, Kondo K, Yoshinari T, Takahashi N, Susuta Y, Kuki H. Efficacy and safety of canagliflozin monotherapy in Japanese patients with type 2 diabetes inadequately controlled with diet and exercise: a 24-week, randomized, double-blind, placebo-controlled, phase III study. Expert Opin Pharmacother 2014;15:1501-15.

74. Ji L, Ma J, Li H, Mansfield TA, T’joen CL, Iqbal N, Ptaszynska A, List JF. Dapagliflozin as monotherapy in drug-naive Asian patients with type 2 diabetes mellitus: a randomized, blinded, prospective phase III study. Clin Ther 2014;36:84-100.

75. Kadowaki T, Haneda M, Inagaki N, Terauchi Y, Taniguchi A, Koiwai K, Rattunde H, Woerle HJ, Broedl UC. Efficacy and safety of empagliflozin monotherapy for 52 weeks in Japanese patients with type 2 diabetes: a randomized, double-blind, parallel-group study. Adv Ther 2015;32:306-18.

76. Lu CH, Min KW, Chuang LM, Kokubo S, Yoshida S, Cha BS. Efficacy, safety, and tolerability of ipragliflozin in Asian patients with type 2 diabetes mellitus and inadequate glycemic control with metformin: results of a phase 3 randomized, placebo-controlled, double-blind, multicenter trial. J Diabetes Investig 2016;7:366-73.

77. Seino Y, Sasaki T, Fukatsu A, Imazeki H, Ochiai H, Sakai S. Efficacy and safety of luseogliflozin added to insulin therapy in Japanese patients with type 2 diabetes: a multicenter, 52-week, clinical study with a 16-week, double-blind period and a 36week, open-label period. Curr Med Res Opin 2018;34:981-94.

78. Yang W, Han P, Min KW, Wang B, Mansfield T, T'Joen C, Iqbal N, Johnsson E, Ptaszynska A. Efficacy and safety of dapagliflozin in Asian patients with type 2 diabetes after metformin failure: a randomized controlled trial. J Diabetes 2016;8: 796-808.

79. Inagaki N, Kondo K, Yoshinari T, Maruyama N, Susuta Y, Kuki H. Efficacy and safety of canagliflozin in Japanese patients with type 2 diabetes: a randomized, double-blind, placebo-controlled, 12-week study. Diabetes Obes Metab 2013;15: 1136-45.

80. Kaku K, Inoue S, Matsuoka O, Kiyosue A, Azuma H, Hayashi N, Tokudome T, Langkilde AM, Parikh S. Efficacy and safety of dapagliflozin as a monotherapy for type 2 diabetes mellitus in Japanese patients with inadequate glycaemic control: a 
phase II multicentre, randomized, double-blind, placebo-controlled trial. Diabetes Obes Metab 2013;15:432-40.

81. Kaku K, Kiyosue A, Inoue S, Ueda N, Tokudome T, Yang J, Langkilde AM. Efficacy and safety of dapagliflozin monotherapy in Japanese patients with type 2 diabetes inadequately controlled by diet and exercise. Diabetes Obes Metab 2014;16: 1102-10.

82. Kadowaki T, Haneda M, Inagaki N, Terauchi Y, Taniguchi A, Koiwai K, Rattunde H, Woerle HJ, Broedl UC. Empagliflozin monotherapy in Japanese patients with type 2 diabetes mellitus: a randomized, 12-week, double-blind, placebo-controlled, phase II trial. Adv Ther 2014;31:621-38.

83. Kashiwagi A, Kazuta K, Takinami Y, Yoshida S, Utsuno A, Nagase I. Ipragliflozin improves glycemic control in Japanese patients with type 2 diabetes mellitus: the BRIGHTEN study. Diabetol Int 2015;6:8-18.

84. Hirose S, Nakajima S, Iwahashi Y, Seo A, Takahashi T, Tamori Y. Impact of the 8-week administration of tofogliflozin for glycemic control and body composition in Japanese patients with type 2 diabetes mellitus. Intern Med 2016;55:3239-45.

85. Ji L, Han P, Liu Y, Yang G, Dieu van NK, Vijapurkar U, Qiu R, Meininger G. Canagliflozin in Asian patients with type 2 diabetes on metformin alone or metformin in combination with sulphonylurea. Diabetes Obes Metab 2015;17:23-31.

86. Kadowaki T, Inagaki N, Kondo K, Nishimura K, Kaneko G, Maruyama N, Nakanishi N, Iijima H, Watanabe Y, Gouda M. Efficacy and safety of canagliflozin as add-on therapy to teneligliptin in Japanese patients with type 2 diabetes mellitus: results of a 24-week, randomized, double-blind, placebo-controlled trial. Diabetes Obes Metab 2017;19:874-82.

87. Kadowaki T, Inagaki N, Kondo K, Nishimura K, Kaneko G, Maruyama N, Nakanishi N, Watanabe Y, Gouda M, Iijima H. Long-term safety and efficacy of canagliflozin as add-on therapy to teneligliptin in Japanese patients with type 2 diabetes. Diabetes Obes Metab 2018;20:77-84.

88. Araki E, Tanizawa Y, Tanaka Y, Taniguchi A, Koiwai K, Kim G, Salsali A, Woerle HJ, Broedl UC. Long-term treatment with empagliflozin as add-on to oral antidiabetes therapy in Japanese patients with type 2 diabetes mellitus. Diabetes Obes Metab 2015;17:665-74.

89. Kashiwagi A, Kazuta K, Goto K, Yoshida S, Ueyama E, Utsuno A. Ipragliflozin in combination with metformin for the treatment of Japanese patients with type 2 diabetes: ILLUMINATE, a randomized, double-blind, placebo-controlled study. Diabetes Obes Metab 2015;17:304-8.
90. Kashiwagi A, Shiga T, Akiyama N, Kazuta K, Utsuno A, Yoshida S, Ueyama E. Efficacy and safety of ipragliflozin as an add-on to pioglitazone in Japanese patients with inadequately controlled type 2 diabetes: a randomized, double-blind, placebo-controlled study (the SPOTLIGHT study). Diabetol Int 2015;6:104-16.

91. Kashiwagi A, Akiyama N, Shiga T, Kazuta K, Utsuno A, Yoshida S, Ueyama E. Efficacy and safety of ipragliflozin as an add-on to a sulfonylurea in Japanese patients with inadequately controlled type 2 diabetes: results of the randomized, placebo-controlled, double-blind, phase III EMIT study. Diabetol Int 2015;6:125-38.

92. Ikeda S, Takano Y, Cynshi O, Tanaka R, Christ AD, Boerlin V, Beyer U, Beck A, Ciorciaro C, Meyer M, Kadowaki T. A novel and selective sodium-glucose cotransporter-2 inhibitor, tofogliflozin, improves glycaemic control and lowers body weight in patients with type 2 diabetes mellitus. Diabetes Obes Metab 2015;17:984-93.

93. Inagaki N, Harashima S, Maruyama N, Kawaguchi Y, Goda M, Iijima $\mathrm{H}$. Efficacy and safety of canagliflozin in combination with insulin: a double-blind, randomized, placebo-controlled study in Japanese patients with type 2 diabetes mellitus. Cardiovasc Diabetol 2016;15:89.

94. Inagaki N, Harashima SI, Kaku K, Kondo K, Maruyama N, Otsuka M, Kawaguchi Y, Iijima H. Long-term efficacy and safety of canagliflozin in combination with insulin in Japanese patients with type 2 diabetes mellitus. Diabetes Obes Metab 2018;20:812-20.

95. Yang W, Ma J, Li Y, Li Y, Zhou Z, Kim JH, Zhao J, Ptaszynska A. Dapagliflozin as add-on therapy in Asian patients with type 2 diabetes inadequately controlled on insulin with or without oral antihyperglycemic drugs: a randomized controlled trial. J Diabetes 2018;10:589-99.

96. Ishihara H, Yamaguchi S, Nakao I, Okitsu A, Asahina S. Efficacy and safety of ipragliflozin as add-on therapy to insulin in Japanese patients with type 2 diabetes mellitus (IOLITE): a multi-centre, randomized, placebo-controlled, double-blind study. Diabetes Obes Metab 2016;18:1207-16.

97. Terauchi Y, Tamura M, Senda M, Gunji R, Kaku K. Efficacy and safety of tofogliflozin in Japanese patients with type 2 diabetes mellitus with inadequate glycaemic control on insulin therapy (J-STEP/INS): results of a 16-week randomized, double-blind, placebo-controlled multicentre trial. Diabetes Obes Metab 2017;19:1397-407.

98. Terauchi Y, Tamura M, Senda M, Gunji R, Kaku K. Long-term 
safety and efficacy of tofogliflozin as add-on to insulin in patients with type 2 diabetes: results from a 52 -week, multicentre, randomized, double-blind, open-label extension, phase 4 study in Japan (J-STEP/INS). Diabetes Obes Metab 2018;20: 1176-85.

99. Suzuki K, Mitsuma Y, Sato T, Anraku T, Hatta M. Comparison of combined tofogliflozin and glargine, tofogliflozin added to insulin, and insulin dose-increase therapy in uncontrolled type 2 diabetes. J Clin Med Res 2016;8:805-14.

100. John M, Cerdas S, Violante R, Deerochanawong C, Hassanein M, Slee A, Canovatchel W, Hamilton G. Efficacy and safety of canagliflozin in patients with type 2 diabetes mellitus living in hot climates. Int J Clin Pract 2016;70:775-85.

101. Yang W, Ji L, Zhou Z, Cain VA, Johnsson KM, Sjostrom CD. Efficacy and safety of dapagliflozin in Asian patients: a pooled analysis. J Diabetes 2017;9:787-99.

102. Yoon KH, Nishimura R, Lee J, Crowe S, Salsali A, Hach T, Woerle HJ. Efficacy and safety of empagliflozin in patients with type 2 diabetes from Asian countries: pooled data from four phase III trials. Diabetes Obes Metab 2016;18:1045-9.

103. Prasanna Kumar KM, Mohan V, Sethi B, Gandhi P, Bantwal G, Xie J, Meininger G, Qiu R. Efficacy and safety of canagliflozin in patients with type 2 diabetes mellitus from India. Indian J Endocrinol Metab 2016;20:372-80.

104. Seino Y, Inagaki N, Haneda M, Kaku K, Sasaki T, Fukatsu A, Ubukata M, Sakai S, Samukawa Y. Efficacy and safety of luseogliflozin added to various oral antidiabetic drugs in Japanese patients with type 2 diabetes mellitus. J Diabetes Investig 2015;6:443-53.

105. Utsunomiya K, Shimmoto N, Senda M, Kurihara Y, Gunji R, Fujii S, Kakiuchi S, Fujiwara H, Kameda H, Tamura M, Kaku K. Safety and effectiveness of tofogliflozin in elderly Japanese patients with type 2 diabetes mellitus: a post-marketing study (J-STEP/EL Study). J Diabetes Investig 2017;8:766-75.

106. Nakamura I, Maegawa H, Tobe K, Tabuchi H, Uno S. Safety and efficacy of ipragliflozin in Japanese patients with type 2 diabetes in real-world clinical practice: interim results of the STELLA-LONG TERM post-marketing surveillance study. Expert Opin Pharmacother 2018;19:189-201.

107. Musso G, Gambino R, Cassader M, Pagano G. A novel approach to control hyperglycemia in type 2 diabetes: sodium glucose co-transport (SGLT) inhibitors: systematic review and meta-analysis of randomized trials. Ann Med 2012;44:375-93.

108. Kaku K, Maegawa H, Tanizawa Y, Kiyosue A, Ide Y, Tokudome T, Hoshino Y, Yang J, Langkilde AM. Dapagliflozin as monotherapy or combination therapy in Japanese patients with type 2 diabetes: an open-label study. Diabetes Ther 2014; 5:415-33.

109. Ohta A, Kato H, Ishii S, Sasaki Y, Nakamura Y, Nakagawa T, Nagai Y, Tanaka Y. Ipragliflozin, a sodium glucose co-transporter 2 inhibitor, reduces intrahepatic lipid content and abdominal visceral fat volume in patients with type 2 diabetes. Expert Opin Pharmacother 2017;18:1433-8.

110. Nishio SI, Sekido T, Ohkubo Y, Oiwa A, Komatsu M. Longterm effects of ipragliflozin on adipose tissue in Japanese patients with obese type 2 diabetes. Shinshu Med J 2018;66:2937.

111. Koshizaka M, Ishikawa K, Ishibashi R, Maezawa Y, Sakamoto K, Uchida D, Nakamura S, Yamaga M, Yokoh H, Kobayashi A, Onishi S, Kobayashi K, Ogino J, Hashimoto N, Tokuyama H, Shimada F, Ohara E, Ishikawa T, Shoji M, Ide S, Ide K, Baba Y, Hattori A, Kitamoto T, Horikoshi T, Shimofusa R, Takahashi S, Nagashima K, Sato Y, Takemoto M, Newby LK, Yokote K; PRIME-V Study Group. Comparing the effects of ipragliflozin versus metformin on visceral fat reduction and metabolic dysfunction in Japanese patients with type 2 diabetes treated with sitagliptin: a prospective, multicentre, open-label, blindedendpoint, randomized controlled study (PRIME-V study). Diabetes Obes Metab 2019;21:1990-5.

112. Yamamoto C, Miyoshi H, Ono K, Sugawara H, Kameda R, Ichiyama M, Yamamoto K, Nomoto H, Nakamura A, Atsumi T. Ipragliflozin effectively reduced visceral fat in Japanese patients with type 2 diabetes under adequate diet therapy. Endocr J 2016;63:589-96.

113. Sugiyama S, Jinnouchi H, Kurinami N, Hieshima K, Yoshida A, Jinnouchi K, Nishimura H, Suzuki T, Miyamoto F, Kajiwara $\mathrm{K}$, Jinnouchi T. Dapagliflozin reduces fat mass without affecting muscle mass in type 2 diabetes. J Atheroscler Thromb 2018;25:467-76.

114. Okamoto A, Yokokawa H, Sanada H, Naito T. Changes in levels of biomarkers associated with adipocyte function and insulin and glucagon kinetics during treatment with dapagliflozin among obese type 2 diabetes mellitus patients. Drugs R D 2016;16:255-61.

115. Roh E, Kim KM, Park KS, Kim YJ, Chun EJ, Choi SH, Park KS, Jang HC, Lim S. Comparison of pancreatic volume and fat amount linked with glucose homeostasis between healthy Caucasians and Koreans. Diabetes Obes Metab 2018;20:264252.

116. Snel M, Jonker JT, Schoones J, Lamb H, de Roos A, Pijl H, 
Smit JW, Meinders AE, Jazet IM. Ectopic fat and insulin resistance: pathophysiology and effect of diet and lifestyle interventions. Int J Endocrinol 2012;2012:983814.

117. Kuchay MS, Krishan S, Mishra SK, Farooqui KJ, Singh MK, Wasir JS, Bansal B, Kaur P, Jevalikar G, Gill HK, Choudhary NS, Mithal A. Effect of empagliflozin on liver fat in patients with type 2 diabetes and nonalcoholic fatty liver disease: a randomized controlled trial (E-LIFT trial). Diabetes Care 2018; 41:1801-8.

118. Ito D, Shimizu S, Inoue K, Saito D, Yanagisawa M, Inukai K, Akiyama Y, Morimoto Y, Noda M, Shimada A. Comparison of ipragliflozin and pioglitazone effects on nonalcoholic fatty liver disease in patients with type 2 diabetes: a randomized, 24-week, open-label, active-controlled trial. Diabetes Care 2017;40:1364-72.

119. Bouchi R, Terashima M, Sasahara Y, Asakawa M, Fukuda T, Takeuchi T, Nakano Y, Murakami M, Minami I, Izumiyama $\mathrm{H}$, Hashimoto K, Yoshimoto T, Ogawa Y. Luseogliflozin reduces epicardial fat accumulation in patients with type 2 diabetes: a pilot study. Cardiovasc Diabetol 2017;16:32.

120. Fukuda T, Bouchi R, Terashima M, Sasahara Y, Asakawa M, Takeuchi T, Nakano Y, Murakami M, Minami I, Izumiyama $\mathrm{H}$, Hashimoto K, Yoshimoto T, Ogawa Y. Ipragliflozin reduces epicardial fat accumulation in non-obese type 2 diabetic patients with visceral obesity: a pilot study. Diabetes Ther 2017;8: 851-61.

121. Grandy S, Hashemi M, Langkilde AM, Parikh S, Sjostrom CD. Changes in weight loss-related quality of life among type 2 diabetes mellitus patients treated with dapagliflozin. Diabetes Obes Metab 2014;16:645-50.

122. Nakajima H, Okada S, Mohri T, Kanda E, Inaba N, Hirasawa Y, Seino H, Kuroda H, Hiyoshi T, Niiya T, Ishii H. Dapagliflozin improves treatment satisfaction in overweight patients with type 2 diabetes mellitus: a patient reported outcome study (PRO study). Diabetol Metab Syndr 2018;10:11.

123. Cai J, Delahanty LM, Akapame S, Slee A, Traina S. Impact of canagliflozin treatment on health-related quality of life among people with type 2 diabetes mellitus: a pooled analysis of patient-reported outcomes from randomized controlled trials. Patient 2018;11:341-52.

124. Look AHEAD Research Group, Wing RR, Bolin P, Brancati FL, Bray GA, Clark JM, Coday M, Crow RS, Curtis JM, Egan CM, Espeland MA, Evans M, Foreyt JP, Ghazarian S, Gregg EW, Harrison B, Hazuda HP, Hill JO, Horton ES, Hubbard VS, Jakicic JM, Jeffery RW, Johnson KC, Kahn SE, Kitabchi AE,
Knowler WC, Lewis CE, Maschak-Carey BJ, Montez MG, Murillo A, Nathan DM, Patricio J, Peters A, Pi-Sunyer X, Pownall H, Reboussin D, Regensteiner JG, Rickman AD, Ryan DH, Safford M, Wadden TA, Wagenknecht LE, West DS, Williamson DF, Yanovski SZ. Cardiovascular effects of intensive lifestyle intervention in type 2 diabetes. N Engl J Med 2013; 369:145-54.

125. Look AHEAD Research Group, Gregg EW, Jakicic JM, Blackburn G, Bloomquist P, Bray GA, Clark JM, Coday M, Curtis JM, Egan C, Evans M, Foreyt J, Foster G, Hazuda HP, Hill JO, Horton ES, Hubbard VS, Jeffery RW, Johnson KC, Kitabchi AE, Knowler WC, Kriska A, Lang W, Lewis CE, Montez MG, Nathan DM, Neiberg RH, Patricio J, Peters A, Pi-Sunyer X, Pownall H, Redmon B, Regensteiner J, Rejeski J, Ribisl PM, Safford M, Stewart K, Trence D, Wadden TA, Wing RR, Yanovski SZ. Association of the magnitude of weight loss and changes in physical fitness with long-term cardiovascular disease outcomes in overweight or obese people with type 2 diabetes: a post-hoc analysis of the Look AHEAD randomised clinical trial. Lancet Diabetes Endocrinol 2016;4:913-21.

126. Faulconbridge LF, Wadden TA, Rubin RR, Wing RR, Walkup MP, Fabricatore AN, Coday M, Van Dorsten B, Mount DL, Ewing LJ; Look AHEAD Research Group. One-year changes in symptoms of depression and weight in overweight/obese individuals with type 2 diabetes in the Look AHEAD study. Obesity (Silver Spring) 2012;20:783-93.

127. Foster GD, Borradaile KE, Sanders MH, Millman R, Zammit G, Newman AB, Wadden TA, Kelley D, Wing RR, Pi-Sunyer FX, Reboussin D, Kuna ST; Sleep AHEAD Research Group of Look AHEAD Research Group. A randomized study on the effect of weight loss on obstructive sleep apnea among obese patients with type 2 diabetes: the Sleep AHEAD study. Arch Intern Med 2009;169:1619-26.

128. Foy CG, Lewis CE, Hairston KG, Miller GD, Lang W, Jakicic JM, Rejeski WJ, Ribisl PM, Walkup MP, Wagenknecht LE; Look AHEAD Research Group. Intensive lifestyle intervention improves physical function among obese adults with knee pain: findings from the Look AHEAD trial. Obesity (Silver Spring) 2011;19:83-93.

129. Phelan S, Kanaya AM, Subak LL, Hogan PE, Espeland MA, Wing RR, Burgio KL, DiLillo V, Gorin AA, West DS, Brown JS; Look AHEAD Research Group. Weight loss prevents urinary incontinence in women with type 2 diabetes: results from the Look AHEAD trial. J Urol 2012;187:939-44.

130. Rejeski WJ, Ip EH, Bertoni AG, Bray GA, Evans G, Gregg EW, 
Zhang Q; Look AHEAD Research Group. Lifestyle change and mobility in obese adults with type 2 diabetes. $\mathrm{N}$ Engl J Med 2012;366:1209-17.

131. Williamson DA, Rejeski J, Lang W, van Dorsten B, Fabricatore AN, Toledo K; Look AHEAD Research Group. Impact of a weight management program on health-related quality of life in overweight adults with type 2 diabetes. Arch Intern Med 2009;169:163-71.

132. Look AHEAD Research Group. Effect of a long-term behavioural weight loss intervention on nephropathy in overweight or obese adults with type 2 diabetes: a secondary analysis of the Look AHEAD randomised clinical trial. Lancet Diabetes Endocrinol 2014;2:801-9.

133. Aldekhail NM, Logue J, McLoone P, Morrison DS. Effect of orlistat on glycaemic control in overweight and obese patients with type 2 diabetes mellitus: a systematic review and metaanalysis of randomized controlled trials. Obes Rev 2015;16: 1071-80.

134. Puzziferri N, Roshek TB 3rd, Mayo HG, Gallagher R, Belle $\mathrm{SH}$, Livingston EH. Long-term follow-up after bariatric surgery: a systematic review. JAMA 2014;312:934-42.

135. Zhou YH, Ma XQ, Wu C, Lu J, Zhang SS, Guo J, Wu SQ, Ye XF, Xu JF, He J. Effect of anti-obesity drug on cardiovascular risk factors: a systematic review and meta-analysis of randomized controlled trials. PLoS One 2012;7:e39062.

136. Ferrannini G, Hach T, Crowe S, Sanghvi A, Hall KD, Ferrannini E. Energy balance after sodium-glucose cotransporter 2 inhibition. Diabetes Care 2015;38:1730-5.

137. Ferrannini E, Muscelli E, Frascerra S, Baldi S, Mari A, Heise T, Broedl UC, Woerle HJ. Metabolic response to sodium-glucose cotransporter 2 inhibition in type 2 diabetic patients. J Clin Invest 2014;124:499-508.

138. Daniele G, Xiong J, Solis-Herrera C, Merovci A, Eldor R, Tripathy D, DeFronzo RA, Norton L, Abdul-Ghani M. Dapagliflozin enhances fat oxidation and ketone production in patients with type 2 diabetes. Diabetes Care 2016;39:2036-41.

139. Ferrannini E, Mark M, Mayoux E. CV protection in the EM-
PA-REG OUTCOME trial: a "thrifty substrate" hypothesis. Diabetes Care 2016;39:1108-14.

140. Bonner C, Kerr-Conte J, Gmyr V, Queniat G, Moerman E, Thevenet J, Beaucamps C, Delalleau N, Popescu I, Malaisse WJ, Sener A, Deprez B, Abderrahmani A, Staels B, Pattou F. Inhibition of the glucose transporter SGLT2 with dapagliflozin in pancreatic alpha cells triggers glucagon secretion. Nat Med 2015;21:512-7.

141. Lee PC, Ganguly S, Goh SY. Weight loss associated with sodium-glucose cotransporter-2 inhibition: a review of evidence and underlying mechanisms. Obes Rev 2018;19:1630-41.

142. Hawley SA, Ford RJ, Smith BK, Gowans GJ, Mancini SJ, Pitt RD, Day EA, Salt IP, Steinberg GR, Hardie DG. The Na+/glucose cotransporter inhibitor canagliflozin activates AMPK by inhibiting mitochondrial function and increasing cellular AMP levels. Diabetes 2016;65:2784-94.

143. Obata A, Kubota N, Kubota T, Iwamoto M, Sato H, Sakurai Y, Takamoto I, Katsuyama H, Suzuki Y, Fukazawa M, Ikeda S, Iwayama K, Tokuyama K, Ueki K, Kadowaki T. Tofogliflozin improves insulin resistance in skeletal muscle and accelerates lipolysis in adipose tissue in male mice. Endocrinology 2016; 157:1029-42.

144. Xu L, Nagata N, Nagashimada M, Zhuge F, Ni Y, Chen G, Mayoux E, Kaneko S, Ota T. SGLT2 inhibition by empagliflozin promotes fat utilization and browning and attenuates inflammation and insulin resistance by polarizing M2 macrophages in diet-induced obese mice. EBioMedicine 2017;20: $137-49$.

145. Inzucchi SE, Zinman B, Wanner C, Ferrari R, Fitchett D, Hantel S, Espadero RM, Woerle HJ, Broedl UC, Johansen OE. SGLT-2 inhibitors and cardiovascular risk: proposed pathways and review of ongoing outcome trials. Diab Vasc Dis Res 2015; 12:90-100.

146. Kimura G. Diuretic action of sodium-glucose cotransporter 2 inhibitors and its importance in the management of heart failure. Circ J 2016;80:2277-81. 